\title{
Targeting KRAS Mutant Cancers via Combination Treatment: Discovery of a 5-Fluoro-4-(3H)-quinazolinone Aryl Urea pan-RAF Kinase Inhibitor
}

Malcolm P. Huestis, Darlene Dela Cruz, Antonio G. DiPasquale, Matthew R. Durk, Charles Eigenbrot, Paul Gibbons, Alberto Gobbi, Thomas L. Hunsaker, Hank La, Dennis H. Leung, Wendy Liu, Shiva Malek, Mark Merchant, John G. Moffat, Christine S. Muli, Christine J. Orr, Brendan T. Parr, Frances Shanahan, Christopher J.

Sneeringer, Weiru Wang, Ivana Yen, Jianping Yin, Michael Siu,* and Joachim Rudolph*

Genentech, Inc., 1 DNA Way, South San Francisco, California 94080, USA

*To whom correspondence should be addressed:

siu.michael@gene.com; rudolph.joachim@gene.com

Figure S1. Small Molecule X-ray Crystal Structure of 6.

Table S1. Coordinates for Small Molecule X-ray Crystal Structure of 6. 2

Table S2. Kinase Selectivity Panel Data for Compounds 1-6. 10

Table S3. Kinase Selectivity Panel Data for Compounds 6, 7. 11

Figure S2. Small Molecule X-ray Crystal Structure of GNE-0749 (7). 17

Table S4. Coordinates for Small Molecule X-ray Crystal Structure of GNE-0749 (7). 17

Qualitative comparison of the intermolecular interaction networks in the small-molecule crystal structures of compounds 6 and 7

Table S5. Crystallography statistics for Figure 4 (X-ray crystal structure of GNE-0749 (7) bound in a mutant BRAF construct).

Figure S3. Synergistic effects of GNE-0749+cobimetinib in KRAS-mutant cell line, HCT116.

Figure S4. Non-synergistic effects of vemurafenib+cobimetinib in KRAS-mutant cell line, HCT116. 


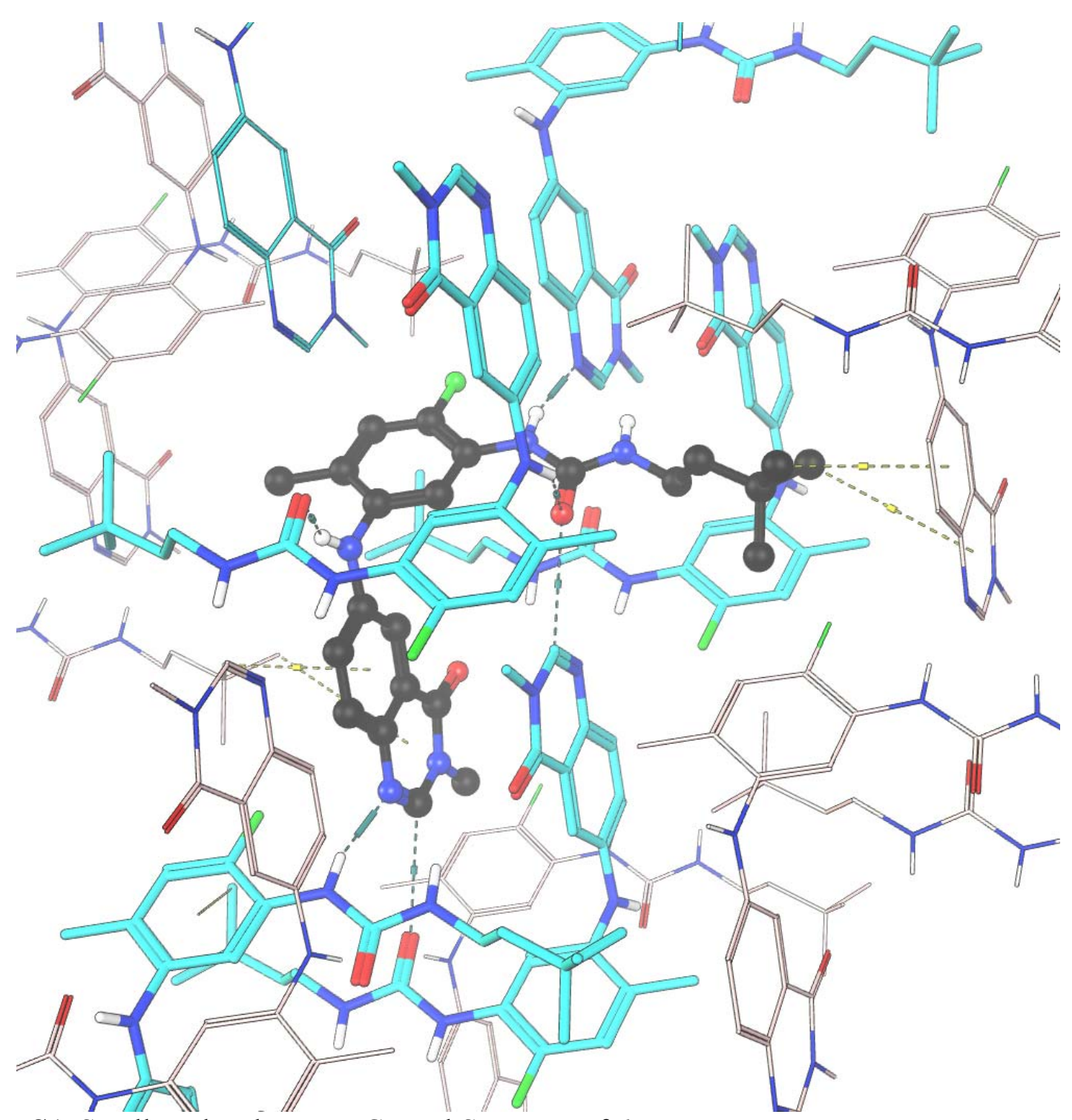

Figure S1. Small Molecule X-ray Crystal Structure of 6.

Table S1. Coordinates for Small Molecule X-ray Crystal Structure of 6.

X-ray quality crystals were grown from a saturated 1,2-dichloroethane/ethanol/methanol solution followed by the slow vapor diffusion of diisopropyl ether to deposit the crystal diffracted. A colorless blade $0.080 \times 0.040 \times 0.020 \mathrm{~mm}$ in size was mounted on a Cryoloop with Paratone oil. Data were collected in a nitrogen gas stream at 100(2) K using phi and omega scans. Crystal-todetector distance was $40 \mathrm{~mm}$ and exposure time was 30 seconds per frame using a scan width of $1.0^{\circ}$. Data collection was $99.8 \%$ complete to $25.000^{\circ}$ in $\theta$. A total of 13647 reflections were collected covering the indices, $-11<=h<=10,-13<=k<=13,-13<=l<=14$. 4095 reflections were found to be symmetry independent, with an $\mathrm{R}_{\text {int }}$ of 0.0631 . Indexing and unit cell refinement indicated a primitive, triclinic lattice. The space group was found to be P -1 (No. 2). The data were integrated using the Bruker SAINT software program and scaled using the SADABS 
software program. Solution by iterative methods (SHELXT) produced a complete heavy-atom phasing model consistent with the proposed structure. All non-hydrogen atoms were refined anisotropically by full-matrix least-squares (SHELXL-2014). All hydrogen atoms were placed using a riding model. Their positions were constrained relative to their parent atom using the appropriate HFIX command in SHELXL-2014.

Crystal data and structure refinement for 6 .

Empirical formula

Formula weight

Temperature

Wavelength

Crystal system

Space group

Unit cell dimensions

Volume

$\mathrm{Z}$

Density (calculated)

Absorption coefficient

$\mathrm{F}(000)$

Crystal size

Theta range for data collection

Index ranges

Reflections collected

Independent reflections

Completeness to theta $=25.000^{\circ}$

Absorption correction

Max. and min. transmission

Refinement method

Data / restraints / parameters

Goodness-of-fit on $\mathrm{F}^{2}$

Final $\mathrm{R}$ indices $[\mathrm{I}>2 \operatorname{sigma}(\mathrm{I})]$

$\mathrm{R}$ indices (all data)

Extinction coefficient

Largest diff. peak and hole

\section{C23 H28 F N5 O2}

425.50

$100(2) \mathrm{K}$

$0.71073 \AA$

Triclinic

P - 1

$\mathrm{a}=9.8391(5) \AA$ $\alpha=72.632(3)^{\circ}$.

$\mathrm{b}=11.1859(6) \AA$ $\beta=89.776(4)^{\circ}$.

$\mathrm{c}=11.8823(7) \AA$ $\gamma=64.843(3)^{\circ}$.

$1118.24(11) \AA^{3}$

2

$1.264 \mathrm{Mg} / \mathrm{m}^{3}$

$0.089 \mathrm{~mm}^{-1}$

452

$0.080 \times 0.040 \times 0.020 \mathrm{~mm}^{3}$

1.814 to $25.371^{\circ}$.

$-11<=\mathrm{h}<=10,-13<=\mathrm{k}<=13,-13<=1<=14$

13647

$4095[\mathrm{R}(\mathrm{int})=0.0631]$

$99.8 \%$

Semi-empirical from equivalents

0.928 and 0.833

Full-matrix least-squares on $\mathrm{F}^{2}$

4095 / 0 / 285

1.031

$\mathrm{R} 1=0.0510, \mathrm{wR} 2=0.1125$

$\mathrm{R} 1=0.1086, \mathrm{wR} 2=0.1375$

$\mathrm{n} / \mathrm{a}$

0.309 and -0.240 e. $\AA^{-3}$ 
Atomic coordinates ( $\left.\mathrm{x} 10^{4}\right)$ and equivalent isotropic displacement parameters $\left(\AA^{2} \mathrm{x} 10^{3}\right)$. U(eq) is defined as one third of the trace of the orthogonalized $\mathrm{U}^{\mathrm{ij}}$ tensor.

\begin{tabular}{|c|c|c|c|c|}
\hline & $\mathrm{x}$ & $\mathrm{y}$ & $\mathrm{z}$ & $\mathrm{U}(\mathrm{eq})$ \\
\hline $\mathrm{C}(1)$ & $8544(3)$ & $8132(2)$ & $3405(2)$ & $19(1)$ \\
\hline$C(2)$ & $6978(3)$ & $8653(3)$ & $3286(2)$ & $21(1)$ \\
\hline$C(3)$ & $6093(3)$ & 9898(3) & $3506(2)$ & $19(1)$ \\
\hline$C(4)$ & 4439(3) & $10452(3)$ & $3328(2)$ & $21(1)$ \\
\hline$C(5)$ & 4429(3) & $12382(3)$ & $3862(2)$ & $24(1)$ \\
\hline$C(6)$ & $6749(3)$ & $10636(3)$ & $3860(2)$ & $21(1)$ \\
\hline$C(7)$ & $8334(3)$ & $10095(3)$ & $3995(2)$ & $24(1)$ \\
\hline $\mathrm{C}(8)$ & $9205(3)$ & $8883(3)$ & $3770(2)$ & $23(1)$ \\
\hline $\mathrm{C}(9)$ & 2023(3) & $12419(3)$ & $3273(2)$ & $31(1)$ \\
\hline$C(10)$ & $8899(3)$ & 6032(3) & $2920(2)$ & $20(1)$ \\
\hline$C(11)$ & 8898(3) & $5883(3)$ & 1793(2) & $24(1)$ \\
\hline$C(12)$ & $8373(3)$ & 4967(3) & $1633(2)$ & $26(1)$ \\
\hline$C(13)$ & 7864(3) & $4265(3)$ & $2547(2)$ & $21(1)$ \\
\hline$C(14)$ & $7809(3)$ & $4418(2)$ & $3671(2)$ & $19(1)$ \\
\hline$C(15)$ & $8366(3)$ & $5316(2)$ & $3837(2)$ & $20(1)$ \\
\hline$C(16)$ & $9441(3)$ & $6682(3)$ & $796(2)$ & $38(1)$ \\
\hline$C(17)$ & 6932(3) & $3775(3)$ & $5620(2)$ & $21(1)$ \\
\hline$C(18)$ & $5900(3)$ & $3000(3)$ & $7407(2)$ & $24(1)$ \\
\hline$C(19)$ & 7201(3) & 2021(3) & $8416(2)$ & $24(1)$ \\
\hline$C(20)$ & $6753(3)$ & 1934(3) & $9668(2)$ & $26(1)$ \\
\hline$C(21)$ & $8185(3)$ & $1035(3)$ & $10584(2)$ & $34(1)$ \\
\hline$C(22)$ & $5678(3)$ & $1247(3)$ & 9916(3) & $46(1)$ \\
\hline$C(23)$ & $5990(3)$ & $3379(3)$ & $9798(2)$ & $39(1)$ \\
\hline $\mathrm{N}(1)$ & $3681(2)$ & $11750(2)$ & $3489(2)$ & $23(1)$ \\
\hline $\mathrm{N}(2)$ & $5892(2)$ & $11890(2)$ & $4068(2)$ & $25(1)$ \\
\hline $\mathrm{N}(3)$ & $9455(2)$ & $6940(2)$ & $3145(2)$ & $22(1)$ \\
\hline $\mathrm{N}(4)$ & $7189(2)$ & $3687(2)$ & $4500(2)$ & $22(1)$ \\
\hline $\mathrm{N}(5)$ & $6306(2)$ & $2966(2)$ & $6233(2)$ & $24(1)$ \\
\hline $\mathrm{O}(1)$ & $3733(2)$ & $9883(2)$ & $3044(2)$ & $28(1)$ \\
\hline $\mathrm{O}(2)$ & $7269(2)$ & $4522(2)$ & $6039(1)$ & $22(1)$ \\
\hline $\mathrm{F}(1)$ & $7346(2)$ & $3377(2)$ & $2356(1)$ & $31(1)$ \\
\hline
\end{tabular}


Bond lengths $[\AA]$ and angles $\left[{ }^{\circ}\right]$.

\begin{tabular}{|c|c|c|c|}
\hline $\mathrm{C}(1)-\mathrm{N}(3)$ & $1.380(3)$ & $\mathrm{C}(14)-\mathrm{N}(4)$ & $1.396(3)$ \\
\hline $\mathrm{C}(1)-\mathrm{C}(2)$ & $1.386(3)$ & $\mathrm{C}(15)-\mathrm{H}(15)$ & 0.9500 \\
\hline $\mathrm{C}(1)-\mathrm{C}(8)$ & $1.415(3)$ & $\mathrm{C}(16)-\mathrm{H}(16 \mathrm{~A})$ & 0.9800 \\
\hline $\mathrm{C}(2)-\mathrm{C}(3)$ & $1.397(3)$ & $\mathrm{C}(16)-\mathrm{H}(16 \mathrm{~B})$ & 0.9800 \\
\hline $\mathrm{C}(2)-\mathrm{H}(2)$ & 0.9500 & $\mathrm{C}(16)-\mathrm{H}(16 \mathrm{C})$ & 0.9800 \\
\hline$C(3)-C(6)$ & $1.392(3)$ & $\mathrm{C}(17)-\mathrm{O}(2)$ & $1.241(3)$ \\
\hline $\mathrm{C}(3)-\mathrm{C}(4)$ & $1.464(3)$ & $\mathrm{C}(17)-\mathrm{N}(5)$ & $1.344(3)$ \\
\hline $\mathrm{C}(4)-\mathrm{O}(1)$ & $1.229(3)$ & $\mathrm{C}(17)-\mathrm{N}(4)$ & $1.378(3)$ \\
\hline $\mathrm{C}(4)-\mathrm{N}(1)$ & $1.394(3)$ & $\mathrm{C}(18)-\mathrm{N}(5)$ & $1.456(3)$ \\
\hline $\mathrm{C}(5)-\mathrm{N}(2)$ & $1.297(3)$ & $C(18)-C(19)$ & $1.521(3)$ \\
\hline $\mathrm{C}(5)-\mathrm{N}(1)$ & $1.367(3)$ & $\mathrm{C}(18)-\mathrm{H}(18 \mathrm{~A})$ & 0.9900 \\
\hline $\mathrm{C}(5)-\mathrm{H}(5)$ & 0.9500 & $\mathrm{C}(18)-\mathrm{H}(18 \mathrm{~B})$ & 0.9900 \\
\hline $\mathrm{C}(6)-\mathrm{N}(2)$ & $1.393(3)$ & $C(19)-C(20)$ & $1.537(3)$ \\
\hline$C(6)-C(7)$ & $1.402(3)$ & $\mathrm{C}(19)-\mathrm{H}(19 \mathrm{~A})$ & 0.9900 \\
\hline$C(7)-C(8)$ & $1.368(3)$ & $\mathrm{C}(19)-\mathrm{H}(19 \mathrm{~B})$ & 0.9900 \\
\hline $\mathrm{C}(7)-\mathrm{H}(7)$ & 0.9500 & $C(20)-C(23)$ & $1.522(4)$ \\
\hline $\mathrm{C}(8)-\mathrm{H}(8)$ & 0.9500 & $C(20)-C(21)$ & $1.528(4)$ \\
\hline $\mathrm{C}(9)-\mathrm{N}(1)$ & $1.462(3)$ & $\mathrm{C}(20)-\mathrm{C}(22)$ & $1.533(3)$ \\
\hline $\mathrm{C}(9)-\mathrm{H}(9 \mathrm{~A})$ & 0.9800 & $\mathrm{C}(21)-\mathrm{H}(21 \mathrm{~A})$ & 0.9800 \\
\hline $\mathrm{C}(9)-\mathrm{H}(9 \mathrm{~B})$ & 0.9800 & $\mathrm{C}(21)-\mathrm{H}(21 \mathrm{~B})$ & 0.9800 \\
\hline $\mathrm{C}(9)-\mathrm{H}(9 \mathrm{C})$ & 0.9800 & $\mathrm{C}(21)-\mathrm{H}(21 \mathrm{C})$ & 0.9800 \\
\hline$C(10)-C(15)$ & $1.391(3)$ & $\mathrm{C}(22)-\mathrm{H}(22 \mathrm{~A})$ & 0.9800 \\
\hline$C(10)-C(11)$ & $1.397(3)$ & $\mathrm{C}(22)-\mathrm{H}(22 \mathrm{~B})$ & 0.9800 \\
\hline $\mathrm{C}(10)-\mathrm{N}(3)$ & $1.427(3)$ & $\mathrm{C}(22)-\mathrm{H}(22 \mathrm{C})$ & 0.9800 \\
\hline$C(11)-C(12)$ & $1.386(3)$ & $\mathrm{C}(23)-\mathrm{H}(23 \mathrm{~A})$ & 0.9800 \\
\hline$C(11)-C(16)$ & $1.503(3)$ & $\mathrm{C}(23)-\mathrm{H}(23 \mathrm{~B})$ & 0.9800 \\
\hline$C(12)-C(13)$ & $1.366(3)$ & $\mathrm{C}(23)-\mathrm{H}(23 \mathrm{C})$ & 0.9800 \\
\hline $\mathrm{C}(12)-\mathrm{H}(12)$ & 0.9500 & $\mathrm{~N}(3)-\mathrm{H}(3)$ & 0.8800 \\
\hline$C(13)-F(1)$ & $1.366(3)$ & $\mathrm{N}(4)-\mathrm{H}(4)$ & 0.8800 \\
\hline$C(13)-C(14)$ & $1.394(3)$ & $\mathrm{N}(5)-\mathrm{H}(5 \mathrm{~A})$ & 0.8800 \\
\hline$C(14)-C(15)$ & $1.392(3)$ & & \\
\hline $\mathrm{N}(3)-\mathrm{C}(1)-\mathrm{C}(2)$ & $122.1(2)$ & $\mathrm{C}(2)-\mathrm{C}(1)-\mathrm{C}(8)$ & $118.2(2)$ \\
\hline $\mathrm{N}(3)-\mathrm{C}(1)-\mathrm{C}(8)$ & $119.7(2)$ & $\mathrm{C}(1)-\mathrm{C}(2)-\mathrm{C}(3)$ & $120.2(2)$ \\
\hline
\end{tabular}




\begin{tabular}{|c|c|}
\hline $\mathrm{C}(1)-\mathrm{C}(2)-\mathrm{H}(2)$ & 119.9 \\
\hline $\mathrm{C}(3)-\mathrm{C}(2)-\mathrm{H}(2)$ & 119.9 \\
\hline$C(6)-C(3)-C(2)$ & $121.4(2)$ \\
\hline$C(6)-C(3)-C(4)$ & $119.6(2)$ \\
\hline $\mathrm{C}(2)-\mathrm{C}(3)-\mathrm{C}(4)$ & $119.0(2)$ \\
\hline $\mathrm{O}(1)-\mathrm{C}(4)-\mathrm{N}(1)$ & $120.9(2)$ \\
\hline $\mathrm{O}(1)-\mathrm{C}(4)-\mathrm{C}(3)$ & $125.5(2)$ \\
\hline $\mathrm{N}(1)-\mathrm{C}(4)-\mathrm{C}(3)$ & $113.5(2)$ \\
\hline $\mathrm{N}(2)-\mathrm{C}(5)-\mathrm{N}(1)$ & $125.2(2)$ \\
\hline $\mathrm{N}(2)-\mathrm{C}(5)-\mathrm{H}(5)$ & 117.4 \\
\hline $\mathrm{N}(1)-\mathrm{C}(5)-\mathrm{H}(5)$ & 117.4 \\
\hline $\mathrm{C}(3)-\mathrm{C}(6)-\mathrm{N}(2)$ & $122.5(2)$ \\
\hline$C(3)-C(6)-C(7)$ & $118.2(2)$ \\
\hline $\mathrm{N}(2)-\mathrm{C}(6)-\mathrm{C}(7)$ & $119.3(2)$ \\
\hline $\mathrm{C}(8)-\mathrm{C}(7)-\mathrm{C}(6)$ & $120.7(2)$ \\
\hline $\mathrm{C}(8)-\mathrm{C}(7)-\mathrm{H}(7)$ & 119.7 \\
\hline $\mathrm{C}(6)-\mathrm{C}(7)-\mathrm{H}(7)$ & 119.7 \\
\hline $\mathrm{C}(7)-\mathrm{C}(8)-\mathrm{C}(1)$ & $121.4(2)$ \\
\hline $\mathrm{C}(7)-\mathrm{C}(8)-\mathrm{H}(8)$ & 119.3 \\
\hline $\mathrm{C}(1)-\mathrm{C}(8)-\mathrm{H}(8)$ & 119.3 \\
\hline $\mathrm{N}(1)-\mathrm{C}(9)-\mathrm{H}(9 \mathrm{~A})$ & 109.5 \\
\hline $\mathrm{N}(1)-\mathrm{C}(9)-\mathrm{H}(9 \mathrm{~B})$ & 109.5 \\
\hline $\mathrm{H}(9 \mathrm{~A})-\mathrm{C}(9)-\mathrm{H}(9 \mathrm{~B})$ & 109.5 \\
\hline $\mathrm{N}(1)-\mathrm{C}(9)-\mathrm{H}(9 \mathrm{C})$ & 109.5 \\
\hline $\mathrm{H}(9 \mathrm{~A})-\mathrm{C}(9)-\mathrm{H}(9 \mathrm{C})$ & 109.5 \\
\hline $\mathrm{H}(9 \mathrm{~B})-\mathrm{C}(9)-\mathrm{H}(9 \mathrm{C})$ & 109.5 \\
\hline $\mathrm{C}(15)-\mathrm{C}(10)-\mathrm{C}(11)$ & $121.6(2)$ \\
\hline $\mathrm{C}(15)-\mathrm{C}(10)-\mathrm{N}(3)$ & $118.7(2)$ \\
\hline $\mathrm{C}(11)-\mathrm{C}(10)-\mathrm{N}(3)$ & $119.7(2)$ \\
\hline $\mathrm{C}(12)-\mathrm{C}(11)-\mathrm{C}(10)$ & $117.2(2)$ \\
\hline $\mathrm{C}(12)-\mathrm{C}(11)-\mathrm{C}(16)$ & $121.3(2)$ \\
\hline $\mathrm{C}(10)-\mathrm{C}(11)-\mathrm{C}(16)$ & $121.5(2)$ \\
\hline $\mathrm{C}(13)-\mathrm{C}(12)-\mathrm{C}(11)$ & $120.4(2)$ \\
\hline $\mathrm{C}(13)-\mathrm{C}(12)-\mathrm{H}(12)$ & 119.8 \\
\hline $\mathrm{C}(11)-\mathrm{C}(12)-\mathrm{H}(12)$ & 119.8 \\
\hline $\mathrm{F}(1)-\mathrm{C}(13)-\mathrm{C}(12)$ & $118.8(2)$ \\
\hline
\end{tabular}

\begin{tabular}{|c|c|}
\hline $\mathrm{F}(1)-\mathrm{C}(13)-\mathrm{C}(14)$ & $117.1(2)$ \\
\hline$C(12)-C(13)-C(14)$ & $124.0(2)$ \\
\hline$C(15)-C(14)-C(13)$ & $115.5(2)$ \\
\hline $\mathrm{C}(15)-\mathrm{C}(14)-\mathrm{N}(4)$ & $126.8(2)$ \\
\hline $\mathrm{C}(13)-\mathrm{C}(14)-\mathrm{N}(4)$ & $117.7(2)$ \\
\hline$C(10)-C(15)-C(14)$ & $121.3(2)$ \\
\hline $\mathrm{C}(10)-\mathrm{C}(15)-\mathrm{H}(15)$ & 119.3 \\
\hline $\mathrm{C}(14)-\mathrm{C}(15)-\mathrm{H}(15)$ & 119.3 \\
\hline $\mathrm{C}(11)-\mathrm{C}(16)-\mathrm{H}(16 \mathrm{~A})$ & 109.5 \\
\hline $\mathrm{C}(11)-\mathrm{C}(16)-\mathrm{H}(16 \mathrm{~B})$ & 109.5 \\
\hline $\mathrm{H}(16 \mathrm{~A})-\mathrm{C}(16)-\mathrm{H}(16 \mathrm{~B})$ & 109.5 \\
\hline $\mathrm{C}(11)-\mathrm{C}(16)-\mathrm{H}(16 \mathrm{C})$ & 109.5 \\
\hline $\mathrm{H}(16 \mathrm{~A})-\mathrm{C}(16)-\mathrm{H}(16 \mathrm{C})$ & 109.5 \\
\hline $\mathrm{H}(16 \mathrm{~B})-\mathrm{C}(16)-\mathrm{H}(16 \mathrm{C})$ & 109.5 \\
\hline $\mathrm{O}(2)-\mathrm{C}(17)-\mathrm{N}(5)$ & $122.5(2)$ \\
\hline $\mathrm{O}(2)-\mathrm{C}(17)-\mathrm{N}(4)$ & $123.1(2)$ \\
\hline $\mathrm{N}(5)-\mathrm{C}(17)-\mathrm{N}(4)$ & $114.4(2)$ \\
\hline $\mathrm{N}(5)-\mathrm{C}(18)-\mathrm{C}(19)$ & $113.7(2)$ \\
\hline $\mathrm{N}(5)-\mathrm{C}(18)-\mathrm{H}(18 \mathrm{~A})$ & 108.8 \\
\hline $\mathrm{C}(19)-\mathrm{C}(18)-\mathrm{H}(18 \mathrm{~A})$ & 108.8 \\
\hline $\mathrm{N}(5)-\mathrm{C}(18)-\mathrm{H}(18 \mathrm{~B})$ & 108.8 \\
\hline $\mathrm{C}(19)-\mathrm{C}(18)-\mathrm{H}(18 \mathrm{~B})$ & 108.8 \\
\hline $\mathrm{H}(18 \mathrm{~A})-\mathrm{C}(18)-\mathrm{H}(18 \mathrm{~B})$ & 107.7 \\
\hline$C(18)-C(19)-C(20)$ & $114.3(2)$ \\
\hline $\mathrm{C}(18)-\mathrm{C}(19)-\mathrm{H}(19 \mathrm{~A})$ & 108.7 \\
\hline $\mathrm{C}(20)-\mathrm{C}(19)-\mathrm{H}(19 \mathrm{~A})$ & 108.7 \\
\hline $\mathrm{C}(18)-\mathrm{C}(19)-\mathrm{H}(19 \mathrm{~B})$ & 108.7 \\
\hline $\mathrm{C}(20)-\mathrm{C}(19)-\mathrm{H}(19 \mathrm{~B})$ & 108.7 \\
\hline $\mathrm{H}(19 \mathrm{~A})-\mathrm{C}(19)-\mathrm{H}(19 \mathrm{~B})$ & 107.6 \\
\hline$C(23)-C(20)-C(21)$ & $108.8(2)$ \\
\hline$C(23)-C(20)-C(22)$ & $109.5(2)$ \\
\hline $\mathrm{C}(21)-\mathrm{C}(20)-\mathrm{C}(22)$ & $108.6(2)$ \\
\hline$C(23)-C(20)-C(19)$ & $111.0(2)$ \\
\hline$C(21)-C(20)-C(19)$ & $108.7(2)$ \\
\hline$C(22)-C(20)-C(19)$ & $110.1(2)$ \\
\hline $\mathrm{C}(20)-\mathrm{C}(21)-\mathrm{H}(21 \mathrm{~A})$ & 109.5 \\
\hline
\end{tabular}




\begin{tabular}{|c|c|}
\hline $\mathrm{C}(20)-\mathrm{C}(21)-\mathrm{H}(21 \mathrm{~B})$ & 109.5 \\
\hline $\mathrm{H}(21 \mathrm{~A})-\mathrm{C}(21)-\mathrm{H}(21 \mathrm{~B})$ & 109.5 \\
\hline $\mathrm{C}(20)-\mathrm{C}(21)-\mathrm{H}(21 \mathrm{C})$ & 109.5 \\
\hline $\mathrm{H}(21 \mathrm{~A})-\mathrm{C}(21)-\mathrm{H}(21 \mathrm{C})$ & 109.5 \\
\hline $\mathrm{H}(21 \mathrm{~B})-\mathrm{C}(21)-\mathrm{H}(21 \mathrm{C})$ & 109.5 \\
\hline $\mathrm{C}(20)-\mathrm{C}(22)-\mathrm{H}(22 \mathrm{~A})$ & 109.5 \\
\hline $\mathrm{C}(20)-\mathrm{C}(22)-\mathrm{H}(22 \mathrm{~B})$ & 109.5 \\
\hline $\mathrm{H}(22 \mathrm{~A})-\mathrm{C}(22)-\mathrm{H}(22 \mathrm{~B})$ & 109.5 \\
\hline $\mathrm{C}(20)-\mathrm{C}(22)-\mathrm{H}(22 \mathrm{C})$ & 109.5 \\
\hline $\mathrm{H}(22 \mathrm{~A})-\mathrm{C}(22)-\mathrm{H}(22 \mathrm{C})$ & 109.5 \\
\hline $\mathrm{H}(22 \mathrm{~B})-\mathrm{C}(22)-\mathrm{H}(22 \mathrm{C})$ & 109.5 \\
\hline $\mathrm{C}(20)-\mathrm{C}(23)-\mathrm{H}(23 \mathrm{~A})$ & 109.5 \\
\hline $\mathrm{C}(20)-\mathrm{C}(23)-\mathrm{H}(23 \mathrm{~B})$ & 109.5 \\
\hline $\mathrm{H}(23 \mathrm{~A})-\mathrm{C}(23)-\mathrm{H}(23 \mathrm{~B})$ & 109.5 \\
\hline $\mathrm{C}(20)-\mathrm{C}(23)-\mathrm{H}(23 \mathrm{C})$ & 109.5 \\
\hline $\mathrm{H}(23 \mathrm{~A})-\mathrm{C}(23)-\mathrm{H}(23 \mathrm{C})$ & 109.5 \\
\hline $\mathrm{H}(23 \mathrm{~B})-\mathrm{C}(23)-\mathrm{H}(23 \mathrm{C})$ & 109.5 \\
\hline $\mathrm{C}(5)-\mathrm{N}(1)-\mathrm{C}(4)$ & $122.5(2)$ \\
\hline $\mathrm{C}(5)-\mathrm{N}(1)-\mathrm{C}(9)$ & $120.0(2)$ \\
\hline $\mathrm{C}(4)-\mathrm{N}(1)-\mathrm{C}(9)$ & $117.5(2)$ \\
\hline $\mathrm{C}(5)-\mathrm{N}(2)-\mathrm{C}(6)$ & $116.5(2)$ \\
\hline $\mathrm{C}(1)-\mathrm{N}(3)-\mathrm{C}(10)$ & $122.86(19)$ \\
\hline $\mathrm{C}(1)-\mathrm{N}(3)-\mathrm{H}(3)$ & 118.6 \\
\hline $\mathrm{C}(10)-\mathrm{N}(3)-\mathrm{H}(3)$ & 118.6 \\
\hline $\mathrm{C}(17)-\mathrm{N}(4)-\mathrm{C}(14)$ & $126.6(2)$ \\
\hline $\mathrm{C}(17)-\mathrm{N}(4)-\mathrm{H}(4)$ & 116.7 \\
\hline $\mathrm{C}(14)-\mathrm{N}(4)-\mathrm{H}(4)$ & 116.7 \\
\hline $\mathrm{C}(17)-\mathrm{N}(5)-\mathrm{C}(18)$ & $120.7(2)$ \\
\hline $\mathrm{C}(17)-\mathrm{N}(5)-\mathrm{H}(5 \mathrm{~A})$ & 119.7 \\
\hline $\mathrm{C}(18)-\mathrm{N}(5)-\mathrm{H}(5 \mathrm{~A})$ & 119.7 \\
\hline
\end{tabular}


Symmetry transformations used to generate equivalent atoms:

Anisotropic displacement parameters $\left(\AA^{2} \times 10^{3}\right)$ for gene427. The anisotropic

displacement factor exponent takes the form: $-2 \pi^{2}\left[\mathrm{~h}^{2} \mathrm{a}^{* 2} \mathrm{U}^{11}+\ldots+2 \mathrm{hk} \mathrm{a} \mathrm{a}^{*} \mathrm{U}^{12}\right]$

\begin{tabular}{|c|c|c|c|c|c|c|}
\hline & $\mathrm{U}^{11}$ & $\mathrm{U}^{22}$ & $\mathrm{U}^{33}$ & $\mathrm{U}^{23}$ & $\mathrm{U}^{13}$ & $\mathrm{U}^{12}$ \\
\hline $\mathrm{C}(1)$ & $23(2)$ & $14(1)$ & $17(1)$ & $-2(1)$ & $0(1)$ & $-8(1)$ \\
\hline$C(2)$ & $26(2)$ & $18(1)$ & $22(1)$ & $-7(1)$ & $0(1)$ & $-12(1)$ \\
\hline$C(3)$ & $24(2)$ & $17(1)$ & $19(1)$ & $-6(1)$ & $2(1)$ & $-12(1)$ \\
\hline $\mathrm{C}(4)$ & $24(2)$ & $19(1)$ & $21(1)$ & $-6(1)$ & $4(1)$ & $-9(1)$ \\
\hline$C(5)$ & $33(2)$ & $17(2)$ & $24(2)$ & $-8(1)$ & $4(1)$ & $-11(1)$ \\
\hline$C(6)$ & $27(2)$ & $16(1)$ & $19(1)$ & $-4(1)$ & $0(1)$ & $-9(1)$ \\
\hline$C(7)$ & $28(2)$ & $22(2)$ & $27(2)$ & $-8(1)$ & $-2(1)$ & $-15(1)$ \\
\hline $\mathrm{C}(8)$ & $21(2)$ & $19(2)$ & $25(1)$ & $-2(1)$ & $-2(1)$ & $-9(1)$ \\
\hline $\mathrm{C}(9)$ & $24(2)$ & $25(2)$ & $41(2)$ & $-12(1)$ & $3(1)$ & $-6(1)$ \\
\hline$C(10)$ & $16(1)$ & $15(1)$ & $29(2)$ & $-9(1)$ & $1(1)$ & $-5(1)$ \\
\hline $\mathrm{C}(11)$ & $24(2)$ & $22(2)$ & $26(2)$ & $-9(1)$ & $4(1)$ & $-11(1)$ \\
\hline$C(12)$ & $30(2)$ & $29(2)$ & $24(2)$ & $-14(1)$ & $6(1)$ & $-13(1)$ \\
\hline $\mathrm{C}(13)$ & $23(2)$ & $16(1)$ & $26(2)$ & $-10(1)$ & $-4(1)$ & $-9(1)$ \\
\hline $\mathrm{C}(14)$ & $16(1)$ & $14(1)$ & $23(1)$ & $-6(1)$ & $-2(1)$ & $-4(1)$ \\
\hline $\mathrm{C}(15)$ & $17(1)$ & $16(1)$ & $22(1)$ & $-7(1)$ & $-3(1)$ & $-4(1)$ \\
\hline$C(16)$ & $48(2)$ & $41(2)$ & $35(2)$ & $-17(2)$ & $15(2)$ & $-28(2)$ \\
\hline $\mathrm{C}(17)$ & $21(2)$ & $15(1)$ & $23(1)$ & $-6(1)$ & $-3(1)$ & $-6(1)$ \\
\hline$C(18)$ & $25(2)$ & $26(2)$ & $23(2)$ & $-9(1)$ & $5(1)$ & $-13(1)$ \\
\hline$C(19)$ & $24(2)$ & $22(2)$ & $26(2)$ & $-8(1)$ & $4(1)$ & $-11(1)$ \\
\hline$C(20)$ & $30(2)$ & $29(2)$ & $23(1)$ & $-8(1)$ & $2(1)$ & $-16(1)$ \\
\hline $\mathrm{C}(21)$ & $37(2)$ & $33(2)$ & $26(2)$ & $-3(1)$ & $0(1)$ & $-15(2)$ \\
\hline $\mathrm{C}(22)$ & $48(2)$ & $67(2)$ & $32(2)$ & $-6(2)$ & $8(2)$ & $-41(2)$ \\
\hline $\mathrm{C}(23)$ & $40(2)$ & $42(2)$ & $31(2)$ & $-18(2)$ & $-3(1)$ & $-9(2)$ \\
\hline $\mathrm{N}(1)$ & $21(1)$ & $16(1)$ & $27(1)$ & $-8(1)$ & $2(1)$ & $-5(1)$ \\
\hline $\mathrm{N}(2)$ & $30(1)$ & $19(1)$ & $27(1)$ & $-11(1)$ & $3(1)$ & $-10(1)$ \\
\hline $\mathrm{N}(3)$ & $17(1)$ & 21(1) & $34(1)$ & $-13(1)$ & $6(1)$ & $-10(1)$ \\
\hline $\mathrm{N}(4)$ & $31(1)$ & $19(1)$ & $23(1)$ & $-10(1)$ & $4(1)$ & $-14(1)$ \\
\hline $\mathrm{N}(5)$ & $35(1)$ & $26(1)$ & $20(1)$ & $-9(1)$ & $2(1)$ & $-20(1)$ \\
\hline $\mathrm{O}(1)$ & $22(1)$ & $25(1)$ & $43(1)$ & $-16(1)$ & $3(1)$ & $-12(1)$ \\
\hline
\end{tabular}




$\begin{array}{lllllll}\mathrm{O}(2) & 23(1) & 19(1) & 29(1) & -12(1) & 1(1) & -11(1) \\ \mathrm{F}(1) & 46(1) & 32(1) & 31(1) & -17(1) & 7(1) & -26(1)\end{array}$

Hydrogen coordinates ( $\left.\times 10^{4}\right)$ and isotropic displacement parameters $\left(\AA^{2} \times 10^{3}\right)$.

\begin{tabular}{|c|c|c|c|c|}
\hline & $\mathrm{x}$ & $\mathrm{y}$ & z & $\mathrm{U}(\mathrm{eq})$ \\
\hline $\mathrm{H}(2)$ & 6506 & 8161 & 3054 & 25 \\
\hline $\mathrm{H}(5)$ & 3831 & 13249 & 3979 & 29 \\
\hline $\mathrm{H}(7)$ & 8807 & 10574 & 4245 & 29 \\
\hline $\mathrm{H}(8)$ & 10277 & 8538 & 3860 & 27 \\
\hline $\mathrm{H}(9 \mathrm{~A})$ & 1633 & 13328 & 3398 & 47 \\
\hline $\mathrm{H}(9 \mathrm{~B})$ & 1645 & 11824 & 3826 & 47 \\
\hline $\mathrm{H}(9 \mathrm{C})$ & 1681 & 12554 & 2452 & 47 \\
\hline $\mathrm{H}(12)$ & 8367 & 4826 & 884 & 32 \\
\hline $\mathrm{H}(15)$ & 8383 & 5443 & 4592 & 23 \\
\hline $\mathrm{H}(16 \mathrm{~A})$ & 8945 & 7675 & 721 & 56 \\
\hline $\mathrm{H}(16 \mathrm{~B})$ & 9188 & 6570 & 49 & 56 \\
\hline $\mathrm{H}(16 \mathrm{C})$ & 10542 & 6323 & 967 & 56 \\
\hline $\mathrm{H}(18 \mathrm{~A})$ & 5050 & 2745 & 7545 & 28 \\
\hline $\mathrm{H}(18 \mathrm{~B})$ & 5538 & 3967 & 7421 & 28 \\
\hline $\mathrm{H}(19 \mathrm{~A})$ & 8007 & 2337 & 8321 & 28 \\
\hline $\mathrm{H}(19 \mathrm{~B})$ & 7632 & 1072 & 8349 & 28 \\
\hline $\mathrm{H}(21 \mathrm{~A})$ & 8690 & 104 & 10503 & 50 \\
\hline $\mathrm{H}(21 \mathrm{~B})$ & 7911 & 951 & 11388 & 50 \\
\hline $\mathrm{H}(21 \mathrm{C})$ & 8873 & 1474 & 10447 & 50 \\
\hline $\mathrm{H}(22 \mathrm{~A})$ & 4727 & 1843 & 9367 & 69 \\
\hline $\mathrm{H}(22 \mathrm{~B})$ & 5465 & 1127 & 10738 & 69 \\
\hline $\mathrm{H}(22 \mathrm{C})$ & 6153 & 332 & 9801 & 69 \\
\hline $\mathrm{H}(23 \mathrm{~A})$ & 6667 & 3827 & 9619 & 59 \\
\hline $\mathrm{H}(23 \mathrm{~B})$ & 5770 & 3290 & 10616 & 59 \\
\hline $\mathrm{H}(23 \mathrm{C})$ & 5041 & 3949 & 9243 & 59 \\
\hline $\mathrm{H}(3)$ & 10421 & 6731 & 3116 & 27 \\
\hline $\mathrm{H}(4)$ & 6937 & 3112 & 4286 & 26 \\
\hline $\mathrm{H}(5 \mathrm{~A})$ & 6140 & 2410 & 5922 & 29 \\
\hline
\end{tabular}


Table S2. Kinase Selectivity Panel Data for Compounds 1-6.

\begin{tabular}{|c|c|c|c|}
\hline Gini Coeff & 0.77 & 0.31 & 0.28 \\
\hline \multirow[t]{2}{*}{ Kinase Count } & 35 & 32 & 32 \\
\hline & ThermoFisher & ThermoFisher & ThermoFisher \\
\hline \multirow[t]{2}{*}{ Source } & $\begin{array}{l}\text { Selectscreen } \\
\text { Compound } 1\end{array}$ & Selectscreen & Selectscreen \\
\hline & @0.1uM & Compound $2 @ 0.1 u M$ & Compound $3 @ 0.1 u M$ \\
\hline Abl & 54.4 & 85.7 & 39.8 \\
\hline B-Raf(V599E) & 100.9 & 82.0 & 99.3 \\
\hline B-Raf(V599E) & 99.5 & 101.0 & 100.7 \\
\hline BRAF & 49.6 & -1.8 & 66.6 \\
\hline BRAF & 99.3 & 101.3 & 101.2 \\
\hline Brk & 24.9 & 35.5 & 1.3 \\
\hline CDK8/cyclinC & 12.5 & & \\
\hline CLK1 & 4.0 & & \\
\hline CSF1R & 70.5 & 85.6 & 81.6 \\
\hline CSK & 82.6 & 73.9 & 42.8 \\
\hline DDR1 & 91.8 & 99.7 & 96.8 \\
\hline EphA2 & 99.7 & 97.3 & 94.9 \\
\hline Fgr & 96.3 & 99.1 & 74.8 \\
\hline Flt1 & 8.8 & 6.6 & 23.9 \\
\hline Flt3 & 50.1 & 2.7 & 63.2 \\
\hline Flt4 & 19.0 & 6.3 & 39.9 \\
\hline JNK2 & 36.5 & & \\
\hline KDR & 37.1 & 8.5 & 78.6 \\
\hline LIMK1 & -3.7 & 40.8 & 66.1 \\
\hline LIMK2 & 4.0 & 40.3 & 45.2 \\
\hline Lck & 94.5 & 100.7 & 88.9 \\
\hline LynB & 100.3 & 99.2 & 91.4 \\
\hline PDGFR_alpha & 66.9 & 97.6 & 33.8 \\
\hline PDGFR_beta & 57.7 & 53.9 & 34.2 \\
\hline RAF1(Y340D,Y341D) & 105.5 & 93.3 & 98.6 \\
\hline RAF1(Y340D,Y341D) & 101.0 & 101.6 & 101.0 \\
\hline RIPK2 & 91.5 & 96.2 & 91.4 \\
\hline Ret & 74.7 & 61.1 & 93.9 \\
\hline Src & 66.8 & 79.4 & 42.8 \\
\hline Srm & 41.1 & 69.8 & 2.5 \\
\hline TNK2 & 0.5 & 23.3 & 9.1 \\
\hline Tie2 & 28.6 & 12.1 & 78.7 \\
\hline Yes & 72.1 & 88.0 & 44.1 \\
\hline ZAK & 65.3 & 95.2 & 68.2 \\
\hline p38_alpha(direct) & 72.5 & 16.2 & 38.3 \\
\hline Gini Coeff & 0.41 & 0.08 & 0.45 \\
\hline Kinase Count & 32 & 32 & 32 \\
\hline & ThermoFisher & ThermoFisher & ThermoFisher \\
\hline Source & Selectscreen & Selectscreen & Selectscreen \\
\hline
\end{tabular}




\begin{tabular}{|c|c|c|c|}
\hline & $\begin{array}{l}\text { Compound } 4 \\
\text { @0.1uM }\end{array}$ & Compound $5 @ 0.1 u M$ & Compound $6 @ 0.1 u M$ \\
\hline Abl & 31.0 & 89.9 & 13.7 \\
\hline B-Raf(V599E) & & 99.1 & 98.8 \\
\hline B-Raf(V599E) & 100.5 & 101.2 & 100.7 \\
\hline BRAF & & 101.1 & 68.3 \\
\hline BRAF & 100.0 & 101.1 & 102.1 \\
\hline Brk & 9.0 & 75.8 & 0.2 \\
\hline CDK8/cyclinC & 1.5 & & \\
\hline CLK1 & 4.5 & & \\
\hline CSF1R & 74.0 & 100.2 & 63.1 \\
\hline CSK & 27.5 & 91.5 & 48.2 \\
\hline DDR1 & 94.5 & 99.0 & 77.7 \\
\hline EphA2 & 81.0 & 94.4 & 51.3 \\
\hline Fgr & 42.0 & 98.8 & 20.3 \\
\hline Flt1 & 7.0 & 60.5 & 1.5 \\
\hline Flt3 & 7.0 & 80.9 & 23.7 \\
\hline Flt4 & 24.0 & 89.9 & 30.4 \\
\hline JNK2 & 77.5 & & \\
\hline KDR & 46.0 & 92.4 & 33.2 \\
\hline LIMK1 & 22.5 & 82.0 & 2.8 \\
\hline LIMK2 & 13.5 & 88.6 & 2.9 \\
\hline Lck & 63.0 & 96.2 & 46.9 \\
\hline LynB & 75.0 & 98.1 & 50.0 \\
\hline PDGFR_alpha & 41.5 & 89.3 & 75.3 \\
\hline PDGFR_beta & 47.5 & 81.7 & 40.4 \\
\hline RAF1(Y3340D,Y341D) & & 99.3 & 100.3 \\
\hline RAF1(Y340D, Y341D) & 101.5 & 101.3 & 101.1 \\
\hline RIPK2 & 94.5 & 100.1 & 71.5 \\
\hline Ret & 81.0 & 96.9 & 89.3 \\
\hline Src & 18.5 & 83.4 & 20.6 \\
\hline Srm & 27.0 & 98.8 & 5.5 \\
\hline TNK2 & -1.0 & 26.1 & 0.4 \\
\hline Tie2 & 31.0 & 92.1 & 0.5 \\
\hline Yes & 13.5 & 84.3 & 17.6 \\
\hline ZAK & 87.0 & 90.3 & 72.7 \\
\hline p38_alpha(direct) & 36.0 & 80.1 & 30.2 \\
\hline
\end{tabular}

Table S3. Kinase Selectivity Panel Data for Compounds 6, 7.

$\begin{array}{lll}\text { Gini Coeff } & 0.64 & 0.72 \\ \text { Kinase Count } & 220 & 225 \\ & \text { ThermoFisher } & \text { ThermoFisher } \\ \text { Source } & \text { Selectscreen } & \text { Selectscreen } \\ & \text { Compound 6 @0.01uM } & \text { GNE-0749 (7) @0.1uM } \\ \text { ACVR1B } & 9.6 & 6.0 \\ \text { ACVR2B } & -0.1 & 11.0 \\ \text { AKT1 } & -1.9 & 0.5 \\ \text { AKT2 } & 9.7 & 6.0 \\ \text { ALK2 } & 1.7 & 1.5\end{array}$




\begin{tabular}{|c|c|c|}
\hline ARK5 & -5.4 & 1.0 \\
\hline ASK1 & -2.9 & 1.5 \\
\hline Abl & 4.7 & 12.6 \\
\hline Aurora_A & 2.2 & 16.0 \\
\hline Aurora_B & 5.7 & 3.5 \\
\hline$A x I$ & 2.0 & -2.5 \\
\hline B-Raf(V599E) & & 100.1 \\
\hline BMPR1A & 3.1 & 4.5 \\
\hline BRAF & 95.0 & 99.6 \\
\hline BTK & -0.5 & 1.5 \\
\hline Blk & -0.9 & 7.0 \\
\hline $\mathrm{Bmx}$ & 0.3 & 2.0 \\
\hline BrSK1 & 5.5 & 3.0 \\
\hline Brk & 5.9 & 2.4 \\
\hline CAMKK1 & -2.8 & -9.5 \\
\hline CAMKK2 & -0.2 & 4.0 \\
\hline CDK1/cyclinB & 4.6 & 2.0 \\
\hline CDK2/cyclinA & 0.3 & -3.5 \\
\hline CDK5/p25 & 2.5 & 8.5 \\
\hline CDK7/cyclinH & 2.6 & -4.5 \\
\hline CDK8/cyclinC & 6.9 & -0.7 \\
\hline CDK9/cyclinT1 & -10.0 & -2.5 \\
\hline CHK1 & -3.7 & 3.5 \\
\hline $\mathrm{CHK} 2$ & 1.7 & 4.5 \\
\hline CK1_alpha1 & -3.0 & 0.0 \\
\hline CK1_delta & 0.3 & 1.0 \\
\hline CK1_epsilon1 & 0.1 & 7.0 \\
\hline CK1_gamma1 & 6.2 & -4.5 \\
\hline CK1_gamma2 & 4.9 & 3.5 \\
\hline CK2_alpha1 & 4.0 & 0.0 \\
\hline CLK $\overline{1}$ & -0.1 & 0.0 \\
\hline CLK2 & 1.6 & 12.0 \\
\hline CLK3 & -0.2 & 7.0 \\
\hline CLK4 & 3.6 & 3.5 \\
\hline CSF1R & 17.7 & 48.8 \\
\hline CSK & 4.1 & 37.7 \\
\hline CaMKI & -3.2 & -4.5 \\
\hline CaMKII_beta & -5.2 & -10.0 \\
\hline CaMKI_delta & 3.7 & -1.5 \\
\hline CamKII_alpha & 4.6 & 3.5 \\
\hline CamKIV & -7.4 & -1.5 \\
\hline DAPK1 & 4.6 & 6.0 \\
\hline DCAMKL2 & 1.5 & 0.0 \\
\hline DDR1 & 18.8 & 70.4 \\
\hline DMPK & 3.1 & -2.0 \\
\hline DNA-PK & 26.7 & 0.5 \\
\hline DRAK1 & 4.5 & 4.0 \\
\hline DYRK1A & 3.3 & 6.0 \\
\hline DYRK3 & 4.5 & -1.5 \\
\hline DYRK4 & -2.6 & 6.5 \\
\hline
\end{tabular}




\begin{tabular}{|c|c|c|}
\hline EGFR & 7.1 & 7.0 \\
\hline EGFR(T790M,L858R) & -6.6 & -5.5 \\
\hline ERK2 & 4.2 & -4.0 \\
\hline \multicolumn{3}{|l|}{ ERN1 } \\
\hline EphA1 & 6.1 & 9.5 \\
\hline EphA2 & & 34.5 \\
\hline EphA3 & -2.9 & 15.5 \\
\hline EphA7 & 7.8 & 2.0 \\
\hline EphA8 & 15.8 & 35.0 \\
\hline EphB1 & 6.1 & 19.0 \\
\hline EphB3 & 0.8 & 6.5 \\
\hline ErbB2 & 5.4 & 0.5 \\
\hline ErbB4 & -9.3 & 9.0 \\
\hline FAK & 3.3 & 4.5 \\
\hline FGFR1 & 12.2 & 2.0 \\
\hline FGFR3 & 10.9 & -2.5 \\
\hline FGFR4 & 2.1 & 5.5 \\
\hline Fes & -8.7 & 7.5 \\
\hline Fgr & 2.6 & 12.8 \\
\hline Flt1 & 5.0 & 3.4 \\
\hline Flt3 & 2.3 & 3.5 \\
\hline Flt4 & 8.8 & 4.6 \\
\hline Frk & 14.5 & 32.5 \\
\hline GRK2 & 1.1 & -0.5 \\
\hline GRK3 & 5.9 & 1.0 \\
\hline GRK5 & 3.6 & 0.5 \\
\hline GRK6 & 10.9 & 2.5 \\
\hline GSK3_alpha & 3.9 & 13.0 \\
\hline GSK3_beta & 2.5 & 5.5 \\
\hline HIPK1- & 2.3 & 2.0 \\
\hline HIPK2 & 1.2 & 1.5 \\
\hline HIPK4 & -3.0 & 3.0 \\
\hline Hyl & 9.3 & -0.5 \\
\hline IGF1R & 4.8 & 8.0 \\
\hline IKK_alpha & 0.6 & -2.0 \\
\hline IKK_beta & -1.0 & 10.0 \\
\hline IKK_epsilon & 3.0 & -0.5 \\
\hline IRAK 1 & 5.7 & 23.0 \\
\hline IRAK4 & 5.8 & 12.0 \\
\hline IRR & 8.0 & 5.5 \\
\hline ITK & 10.9 & 2.5 \\
\hline InsR & 3.3 & 0.0 \\
\hline JAK1 & 0.3 & 3.0 \\
\hline JAK2 & 5.7 & -6.0 \\
\hline JAK3 & 5.9 & 6.0 \\
\hline JNK1_alpha1 & -2.5 & 2.5 \\
\hline JNK2 ${ }^{-}$ & 6.0 & 16.9 \\
\hline JNK3 & -5.5 & 8.0 \\
\hline KDR & 4.0 & 11.3 \\
\hline KHS1 & 2.3 & 20.0 \\
\hline
\end{tabular}




\begin{tabular}{|c|c|c|}
\hline Kit & 10.5 & 6.0 \\
\hline LIMK1 & -6.2 & 0.5 \\
\hline LIMK2 & & -10.6 \\
\hline LRRK2 & -7.5 & -8.0 \\
\hline LTK & 3.1 & 4.5 \\
\hline Lck & 4.7 & 28.8 \\
\hline Lyn & 9.3 & 38.5 \\
\hline LynB & & 50.8 \\
\hline MAP4K4 & 22.5 & 20.5 \\
\hline MAPKAPK2 & 0.1 & 8.5 \\
\hline MAPKAPK3 & -2.4 & 6.0 \\
\hline MARK1 & 6.4 & 0.0 \\
\hline MARK3 & 1.5 & -1.0 \\
\hline MEK1 & 2.9 & -1.0 \\
\hline MEK3 & -4.7 & 0.5 \\
\hline MEKK2 & 3.2 & -3.5 \\
\hline MELK & 8.6 & 13.0 \\
\hline MKK6 & 0.3 & 1.0 \\
\hline MKNK1 & -4.0 & -1.0 \\
\hline MKNK2 & 3.4 & -2.0 \\
\hline MLK1 & 8.8 & 5.5 \\
\hline MLK2 & -3.4 & 2.0 \\
\hline MRCK_alpha & 4.3 & 7.5 \\
\hline MSK1 & 8.9 & 2.0 \\
\hline MSSK1 & 0.6 & 5.0 \\
\hline MST1 & 4.7 & -8.0 \\
\hline MST2 & 9.8 & 4.5 \\
\hline MST3 & 2.6 & 8.0 \\
\hline MST4 & 5.7 & -2.5 \\
\hline MYLK(smMLCK) & 4.1 & 9.0 \\
\hline MYLK3(caMLCK) & 12.4 & 2.0 \\
\hline Mer & 6.6 & 2.5 \\
\hline Met & 4.3 & -5.0 \\
\hline Mink1 & 21.1 & 2.5 \\
\hline MuSK & 25.8 & 10.5 \\
\hline NEK1 & 3.8 & 0.0 \\
\hline NEK4 & 2.5 & -0.5 \\
\hline NEK6 & 8.5 & 4.5 \\
\hline NEK9 & 12.8 & 2.5 \\
\hline NLK & 2.8 & 0.5 \\
\hline PAK1 & 4.4 & 8.0 \\
\hline PAK3 & 10.1 & 7.5 \\
\hline PAK4 & 5.5 & 3.5 \\
\hline PAK6 & 4.5 & 8.0 \\
\hline PASK & 3.8 & 2.0 \\
\hline PDGFR_alpha & 25.0 & 39.2 \\
\hline PDGFR_beta & & 27.8 \\
\hline PDK1(direct) & 1.0 & -14.5 \\
\hline PI3K-A & -6.4 & -10.0 \\
\hline PI3K-G & 7.9 & 6.5 \\
\hline
\end{tabular}




\begin{tabular}{|c|c|c|}
\hline PIM1 & 2.6 & 4.5 \\
\hline PKA & -0.6 & 0.0 \\
\hline PKC_alpha & 10.2 & 4.5 \\
\hline PKC_beta1 & 4.2 & 5.0 \\
\hline PKC_delta & -0.1 & 3.5 \\
\hline PKC_epsilon & -0.3 & 12.0 \\
\hline PKC_eta & 14.7 & 6.0 \\
\hline PKC_theta & 5.8 & 5.5 \\
\hline PKC_zeta & 10.2 & 6.5 \\
\hline PKD1 & 1.0 & 10.5 \\
\hline PKG1_alpha & 1.2 & 6.0 \\
\hline PLK1 & 2.5 & -4.0 \\
\hline PLK2 & 8.8 & -3.0 \\
\hline PLK3 & 12.1 & -3.0 \\
\hline PRAK & 6.2 & 0.0 \\
\hline PRK1 & -11.5 & 3.0 \\
\hline PRKAA1 & -0.3 & 1.5 \\
\hline PhK_gamma1 & 5.7 & -1.5 \\
\hline PhK_gamma2 & 9.1 & -3.5 \\
\hline $\operatorname{PrK} \bar{X}$ & 1.9 & 0.0 \\
\hline RAF1(Y340D,Y341D) & 99.1 & 100.8 \\
\hline RIPK2 & 16.3 & 50.8 \\
\hline ROCK1 & -0.7 & 6.5 \\
\hline ROCK2 & -1.5 & -7.5 \\
\hline RSK1 & 2.0 & 3.5 \\
\hline RSK2 & -0.1 & 8.0 \\
\hline RSK3 & 4.2 & 3.5 \\
\hline Ret & 28.8 & 62.3 \\
\hline Ron & -3.1 & 0.0 \\
\hline Ros & 5.2 & 3.5 \\
\hline Rse & 2.8 & 6.0 \\
\hline SGK1 & -0.1 & 4.0 \\
\hline SGK2 & 0.0 & 5.0 \\
\hline SGK3 & 0.3 & -1.5 \\
\hline SIK2 & 2.0 & 7.0 \\
\hline SLK & -4.5 & 1.0 \\
\hline SPHK1 & -6.3 & 0.0 \\
\hline SRPK1 & 1.4 & 3.0 \\
\hline STK16 & -10.8 & -0.5 \\
\hline STK33 & -6.4 & 1.0 \\
\hline Src & 1.6 & 11.6 \\
\hline Srm & 1.5 & 7.4 \\
\hline Syk & 2.3 & 4.5 \\
\hline TAK1-TAB1 & -3.8 & -3.5 \\
\hline TAO2 & 0.0 & 3.5 \\
\hline TBK1 & -2.6 & 2.0 \\
\hline TEC & 0.4 & -3.0 \\
\hline TGFBR1 & 19.0 & -1.5 \\
\hline TNK2 & -2.3 & 0.4 \\
\hline TSSK1 & 3.9 & 3.0 \\
\hline
\end{tabular}




$\begin{array}{lll}\text { TTK } & 3.3 & 1.5 \\ \text { TXK } & 4.8 & -1.0 \\ \text { TYK2 } & 3.2 & 7.0 \\ \text { Tie2 } & 14.1 & 12.9 \\ \text { Tpl2 } & -2.4 & 5.5 \\ \text { TrkA } & 4.6 & 0.0 \\ \text { TrkB } & 3.6 & 4.0 \\ \text { WEE1 } & -1.4 & -4.0 \\ \text { WNK2 } & 8.1 & 0.0 \\ \text { YSK1 } & 9.7 & -2.0 \\ \text { Yes } & 4.7 & 12.3 \\ \text { ZAK } & 25.8 & 51.1 \\ \text { ZAP-70 } & 9.9 & -8.5 \\ \text { ZIPK } & -7.4 & 5.5 \\ \text { eEF-2K } & 0.8 & 2.5 \\ \text { mTOR } & -0.6 & -9.0 \\ \text { p38_alpha(direct) } & 7.6 & 55.1 \\ \text { p38_beta } & 10.3 & 13.0 \\ \text { p38_delta } & 5.8 & 5.0 \\ \text { p38_gamma } & 5.3 & 0.5 \\ \text { p70S6K } & 5.6 & 2.5 \\ \end{array}$




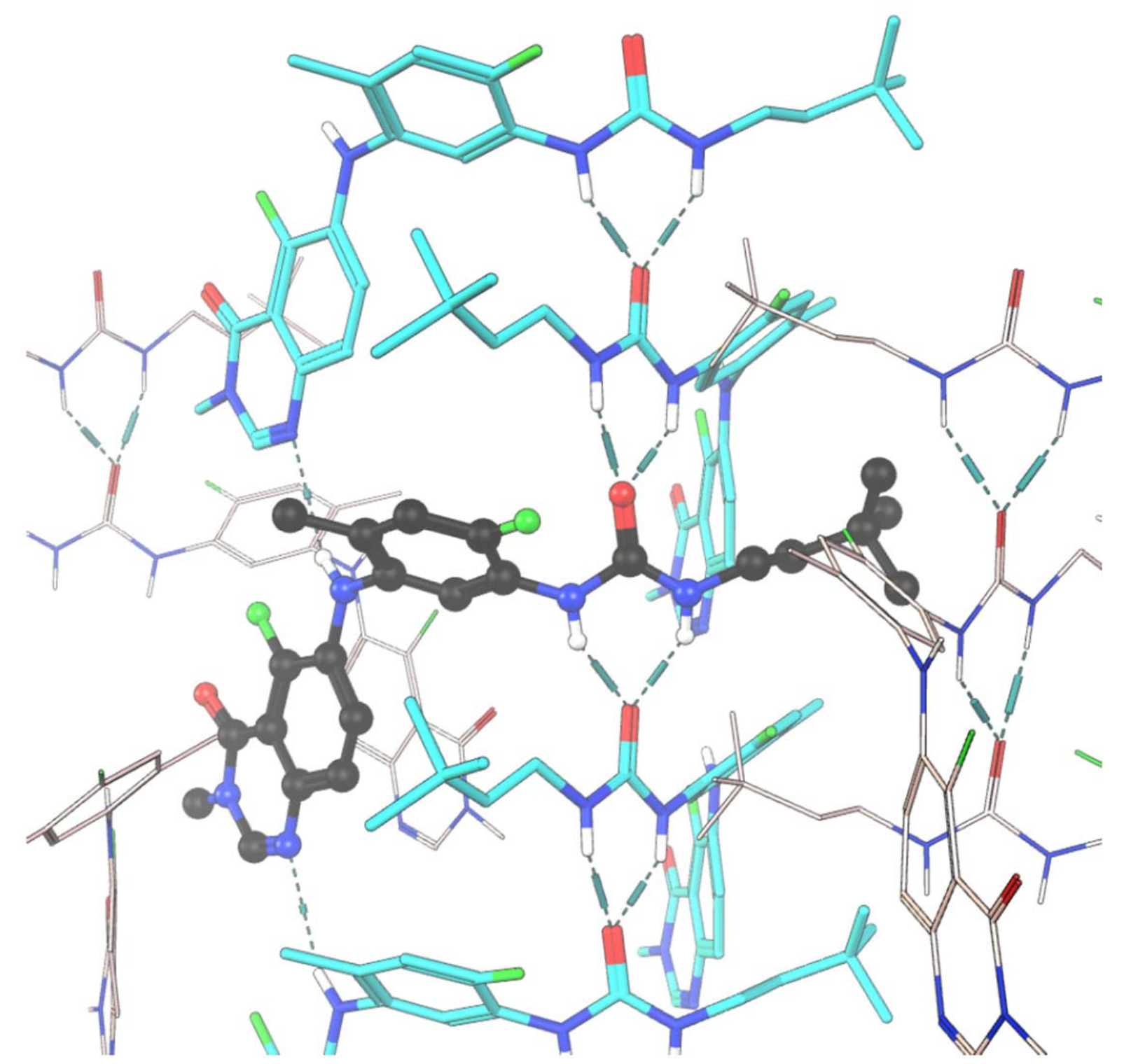

Figure S2. Small Molecule X-ray Crystal Structure of GNE-0749 (7).

Table S4. Coordinates for Small Molecule X-ray Crystal Structure of GNE-0749 (7).

X-ray quality crystals were grown from a saturated 1,2-dichloroethane/ethanol/methanol solution followed by the slow vapor diffusion of heptane to deposit the crystal diffracted. A colorless plate $0.040 \times 0.040 \times 0.020 \mathrm{~mm}$ in size was mounted on a Cryoloop with Paratone oil. Data were collected in a nitrogen gas stream at $100(2) \mathrm{K}$ using and scans. Crystal-to-detector distance was $60 \mathrm{~mm}$ and exposure time was 30 seconds per frame using a scan width of $2.0^{\circ}$. Data collection was $99.5 \%$ complete to $50.000^{\circ}$ in $\theta$. A total of 15275 reflections were collected covering the indices, $-36<=h<=36,-8<=k<=8,-10<=k<=13.4379$ reflections were found to be symmetry independent, with an $\mathrm{R}_{\text {int }}$ of 0.0818 . Indexing and unit cell refinement indicated a $\mathrm{C}$-centered, monoclinic lattice. The space group was found to be $\mathrm{C} 2$ (No. 5). The data were integrated using the Bruker SAINT software program and scaled using the SADABS software program. Solution 
by iterative methods (SHELXT-2014) produced a complete heavy-atom phasing model consistent with the proposed structure. All non-hydrogen atoms were refined isotropically by full-matrix least-squares (SHELXL-2014). All hydrogen atoms were placed using a riding model. Their positions were constrained relative to their parent atom using the appropriate HFIX command in SHELXL-2014.

Crystal data and structure refinement for GNE-0749 (7).

X-ray ID

Sample/notebook ID

Empirical formula

Formula weight

Temperature

Wavelength

Crystal system

Space group

Unit cell dimensions

Volume

Z

Density (calculated)

Absorption coefficient

$\mathrm{F}(000)$

Crystal size

Theta range for data collection

Index ranges

Reflections collected

Independent reflections

Completeness to theta $=50.000^{\circ}$

Absorption correction

Max. and min. transmission

Refinement method

Data / restraints / parameters

Goodness-of-fit on $\mathrm{F}^{2}$

Final $\mathrm{R}$ indices [I $>2 \operatorname{sigma}(\mathrm{I})]$

$\mathrm{R}$ indices (all data)

Absolute structure parameter gene 560

G03070749.1-3

C23 H27 F2 N5 O2

443.49

$100(2) \mathrm{K}$

$1.54178 \AA$

Monoclinic

C 2

$\mathrm{a}=36.459(4) \AA$

$\alpha=90^{\circ}$.

$\mathrm{b}=8.6753(8) \AA$

$\beta=98.333(7)^{\circ}$.

$\mathrm{c}=13.8617(10) \AA$

$\gamma=90^{\circ}$.

4338.1(7) $\AA^{3}$

8

$1.358 \mathrm{Mg} / \mathrm{m}^{3}$

$0.840 \mathrm{~mm}^{-1}$

1872

$0.040 \times 0.040 \times 0.020 \mathrm{~mm}^{3}$

2.450 to $50.465^{\circ}$.

$-36<=\mathrm{h}<=36,-8<=\mathrm{k}<=8,-10<=\mathrm{l}<=13$

15275

$4379[\mathrm{R}(\mathrm{int})=0.0818]$

$99.5 \%$

Semi-empirical from equivalents

0.983 and 0.967

Full-matrix least-squares on $\mathrm{F}^{2}$

4379 / 1 / 269

1.091

$\mathrm{R} 1=0.1629, \mathrm{wR} 2=0.3854$

$\mathrm{R} 1=0.1733, \mathrm{wR} 2=0.3938$

$-0.01(14)$ 
Extinction coefficient

Largest diff. peak and hole
$0.0015(4)$

1.380 and -0.719 e. $\AA^{-3}$

Table 2. Atomic coordinates $\left(\mathrm{x} 10^{4}\right)$ and equivalent isotropic displacement parameters $\left(\AA^{2} \times 10^{3}\right)$. U(eq) is defined as one third of the trace of the orthogonalized $U^{i j}$ tensor.

\begin{tabular}{|c|c|c|c|c|}
\hline & $\mathrm{x}$ & $\mathrm{y}$ & $\mathrm{Z}$ & $\mathrm{U}(\mathrm{eq})$ \\
\hline $\mathrm{C}(1)$ & $4505(7)$ & $2390(30)$ & 3963(19) & $27(6)$ \\
\hline$C(2)$ & $4860(6)$ & $2790(30)$ & $3775(18)$ & $23(6)$ \\
\hline$C(3)$ & $4987(6)$ & $4220(30)$ & $3726(18)$ & $25(6)$ \\
\hline$C(4)$ & $5376(6)$ & $4570(30)$ & $3611(17)$ & $21(6)$ \\
\hline$C(5)$ & $5185(6)$ & $7310(30)$ & $3762(17)$ & $19(5)$ \\
\hline$C(6)$ & $4730(6)$ & $5460(30)$ & $3843(16)$ & $13(5)$ \\
\hline$C(7)$ & $4373(6)$ & $5140(30)$ & 3951(17) & $22(6)$ \\
\hline$C(8)$ & $4279(6)$ & $3590(30)$ & 4094(17) & $23(6)$ \\
\hline $\mathrm{C}(9)$ & $5838(6)$ & $6670(30)$ & $3716(18)$ & $22(6)$ \\
\hline$C(10)$ & $4049(6)$ & $180(30)$ & $4218(17)$ & $24(6)$ \\
\hline $\mathrm{C}(11)$ & $3927(6)$ & $710(30)$ & $5046(18)$ & $23(6)$ \\
\hline$C(12)$ & $3589(5)$ & $140(30)$ & $5292(15)$ & $13(5)$ \\
\hline$C(13)$ & $3387(6)$ & $-880(30)$ & $4618(18)$ & $31(6)$ \\
\hline$C(14)$ & $3533(5)$ & $-1350(30)$ & $3793(17)$ & $20(5)$ \\
\hline$C(15)$ & $3879(6)$ & $-760(30)$ & $3606(16)$ & $20(5)$ \\
\hline$C(16)$ & $4012(6)$ & $-1320(30)$ & $2695(17)$ & $25(6)$ \\
\hline $\mathrm{C}(17)$ & $3389(9)$ & $-370(40)$ & $6800(30)$ & $56(9)$ \\
\hline$C(18)$ & $3217(9)$ & $-860(40)$ & $8450(20)$ & $63(9)$ \\
\hline$C(19)$ & $3155(10)$ & $-150(40)$ & $9270(30)$ & $69(10)$ \\
\hline$C(20)$ & $2973(6)$ & $-930(30)$ & $10065(17)$ & $26(6)$ \\
\hline $\mathrm{C}(21)$ & $2604(10)$ & $-1420(50)$ & $9830(30)$ & $82(11)$ \\
\hline$C(22)$ & $3196(8)$ & $-2390(40)$ & $10390(20)$ & $49(8)$ \\
\hline $\mathrm{C}(23)$ & $3043(9)$ & $100(40)$ & $11070(20)$ & $57(9)$ \\
\hline$C(24)$ & $4529(6)$ & $7310(30)$ & $10625(18)$ & $24(6)$ \\
\hline$C(25)$ & $4880(6)$ & $7630(30)$ & $11100(17)$ & $21(6)$ \\
\hline$C(26)$ & 4997(6) & $9230(30)$ & $11250(16)$ & $14(5)$ \\
\hline $\mathrm{C}(27)$ & $5373(7)$ & $9580(30)$ & $11627(18)$ & $27(6)$ \\
\hline$C(28)$ & $5188(7)$ & $12270(30)$ & $11388(19)$ & $31(7)$ \\
\hline
\end{tabular}




\begin{tabular}{|c|c|c|c|c|}
\hline $\mathrm{C}(29)$ & $4744(5)$ & $10450(20)$ & 10971(15) & $12(5)$ \\
\hline $\mathrm{C}(30)$ & $4386(7)$ & $10020(30)$ & $10514(19)$ & $30(6)$ \\
\hline $\mathrm{C}(31)$ & $4284(7)$ & $8610(30)$ & $10380(20)$ & $42(7)$ \\
\hline $\mathrm{C}(32)$ & $5827(7)$ & $11600(30)$ & $11950(20)$ & $35(7)$ \\
\hline $\mathrm{C}(33)$ & $4088(6)$ & $5180(30)$ & $10035(17)$ & $19(5)$ \\
\hline $\mathrm{C}(34)$ & $3949(7)$ & $5630(30)$ & $9098(19)$ & $30(6)$ \\
\hline$C(35)$ & $3607(6)$ & $5130(30)$ & $8648(18)$ & $26(6)$ \\
\hline$C(36)$ & $3409(5)$ & $4100(30)$ & $9153(15)$ & $14(5)$ \\
\hline$C(37)$ & $3557(6)$ & $3690(30)$ & $10136(16)$ & $22(5)$ \\
\hline $\mathrm{C}(38)$ & $3889(6)$ & $4200(30)$ & $10567(17)$ & $24(6)$ \\
\hline C(39) & $4033(6)$ & $3770(30)$ & $11612(16)$ & $25(6)$ \\
\hline $\mathrm{C}(40)$ & $3286(8)$ & $4660(30)$ & $6950(20)$ & $46(8)$ \\
\hline $\mathrm{C}(41)$ & $2936(8)$ & $4680(40)$ & $5340(20)$ & $51(8)$ \\
\hline $\mathrm{C}(42)$ & $3152(8)$ & $4620(40)$ & $4410(20)$ & $54(9)$ \\
\hline $\mathrm{C}(43)$ & $2943(7)$ & $3950(40)$ & $3470(20)$ & $46(7)$ \\
\hline $\mathrm{C}(44)$ & $2688(11)$ & $2640(50)$ & $3610(30)$ & $83(12)$ \\
\hline $\mathrm{C}(45)$ & 2712(9) & $5240(40)$ & $3110(30)$ & $61(9)$ \\
\hline$C(46)$ & $3173(10)$ & $3640(50)$ & $2770(30)$ & $87(12)$ \\
\hline $\mathrm{N}(1)$ & $5440(5)$ & $6230(20)$ & $3723(14)$ & $24(5)$ \\
\hline $\mathrm{N}(2)$ & $4851(5)$ & $7000(20)$ & $3820(14)$ & $19(5)$ \\
\hline $\mathrm{N}(3)$ & $4423(5)$ & $840(20)$ & $4050(15)$ & $30(5)$ \\
\hline $\mathrm{N}(4)$ & $3452(5)$ & $680(20)$ & $6108(14)$ & $22(5)$ \\
\hline $\mathrm{N}(5)$ & $3259(7)$ & $210(30)$ & $7596(19)$ & $59(7)$ \\
\hline $\mathrm{N}(6)$ & $5460(4)$ & $11140(20)$ & $11670(13)$ & $17(4)$ \\
\hline $\mathrm{N}(7)$ & $4853(5)$ & $11980(20)$ & $11057(14)$ & $18(5)$ \\
\hline $\mathrm{N}(8)$ & $4429(6)$ & $5650(30)$ & $10488(16)$ & $37(6)$ \\
\hline $\mathrm{N}(9)$ & $3450(5)$ & $5560(20)$ & $7709(13)$ & $20(5)$ \\
\hline $\mathrm{N}(10)$ & $3154(7)$ & $5400(30)$ & 6143(19) & $58(7)$ \\
\hline $\mathrm{O}(1)$ & $5617(4)$ & $3700(20)$ & $3519(11)$ & $27(4)$ \\
\hline $\mathrm{O}(2)$ & $3440(5)$ & $-1800(20)$ & $6712(14)$ & $44(5)$ \\
\hline $\mathrm{O}(3)$ & $5620(4)$ & $8680(20)$ & $11945(12)$ & $32(4)$ \\
\hline $\mathrm{O}(4)$ & $3279(5)$ & $3180(20)$ & $7103(15)$ & $53(6)$ \\
\hline $\mathrm{F}(1)$ & $5098(3)$ & $1548(15)$ & $3695(9)$ & $24(3)$ \\
\hline $\mathrm{F}(2)$ & $3078(3)$ & $-1361(17)$ & $4770(10)$ & $35(4)$ \\
\hline $\mathrm{F}(3)$ & $5103(3)$ & $6464(16)$ & $11400(10)$ & $31(3)$ \\
\hline
\end{tabular}


Bond lengths $[\AA]$ and angles $\left[^{\circ}\right]$.

\begin{tabular}{|c|c|c|c|}
\hline $\mathrm{C}(1)-\mathrm{C}(8)$ & $1.36(3)$ & $\mathrm{C}(14)-\mathrm{H}(14)$ & 0.9500 \\
\hline $\mathrm{C}(1)-\mathrm{N}(3)$ & $1.39(3)$ & $C(15)-C(16)$ & $1.50(3)$ \\
\hline$C(1)-C(2)$ & $1.40(3)$ & $\mathrm{C}(16)-\mathrm{H}(16 \mathrm{~A})$ & 0.9800 \\
\hline$C(2)-C(3)$ & $1.33(3)$ & $\mathrm{C}(16)-\mathrm{H}(16 \mathrm{~B})$ & 0.9800 \\
\hline$C(2)-F(1)$ & $1.40(3)$ & $\mathrm{C}(16)-\mathrm{H}(16 \mathrm{C})$ & 0.9800 \\
\hline $\mathrm{C}(3)-\mathrm{C}(6)$ & $1.45(3)$ & $\mathrm{C}(17)-\mathrm{O}(2)$ & $1.27(3)$ \\
\hline $\mathrm{C}(3)-\mathrm{C}(4)$ & $1.48(3)$ & $\mathrm{C}(17)-\mathrm{N}(5)$ & $1.36(4)$ \\
\hline $\mathrm{C}(4)-\mathrm{O}(1)$ & $1.18(3)$ & $\mathrm{C}(17)-\mathrm{N}(4)$ & $1.36(4)$ \\
\hline $\mathrm{C}(4)-\mathrm{N}(1)$ & $1.46(3)$ & $C(18)-C(19)$ & $1.34(5)$ \\
\hline $\mathrm{C}(5)-\mathrm{N}(2)$ & $1.26(3)$ & $\mathrm{C}(18)-\mathrm{N}(5)$ & $1.53(4)$ \\
\hline $\mathrm{C}(5)-\mathrm{N}(1)$ & $1.32(3)$ & $\mathrm{C}(18)-\mathrm{H}(18 \mathrm{~A})$ & 0.9900 \\
\hline $\mathrm{C}(5)-\mathrm{H}(5)$ & 0.9500 & $\mathrm{C}(18)-\mathrm{H}(18 \mathrm{~B})$ & 0.9900 \\
\hline$C(6)-C(7)$ & $1.36(3)$ & $C(19)-C(20)$ & $1.52(4)$ \\
\hline $\mathrm{C}(6)-\mathrm{N}(2)$ & $1.41(3)$ & $\mathrm{C}(19)-\mathrm{H}(19 \mathrm{~A})$ & 0.9900 \\
\hline$C(7)-C(8)$ & $1.40(3)$ & $\mathrm{C}(19)-\mathrm{H}(19 \mathrm{~B})$ & 0.9900 \\
\hline $\mathrm{C}(7)-\mathrm{H}(7)$ & 0.9500 & $C(20)-C(21)$ & $1.40(4)$ \\
\hline $\mathrm{C}(8)-\mathrm{H}(8)$ & 0.9500 & $C(20)-C(22)$ & $1.53(4)$ \\
\hline $\mathrm{C}(9)-\mathrm{N}(1)$ & $1.50(3)$ & $C(20)-C(23)$ & $1.64(4)$ \\
\hline $\mathrm{C}(9)-\mathrm{H}(9 \mathrm{~A})$ & 0.9800 & $\mathrm{C}(21)-\mathrm{H}(21 \mathrm{~A})$ & 0.9800 \\
\hline C(9)-H(9B) & 0.9800 & $\mathrm{C}(21)-\mathrm{H}(21 \mathrm{~B})$ & 0.9800 \\
\hline $\mathrm{C}(9)-\mathrm{H}(9 \mathrm{C})$ & 0.9800 & $\mathrm{C}(21)-\mathrm{H}(21 \mathrm{C})$ & 0.9800 \\
\hline$C(10)-C(15)$ & $1.27(3)$ & $\mathrm{C}(22)-\mathrm{H}(22 \mathrm{~A})$ & 0.9800 \\
\hline$C(10)-C(11)$ & $1.37(3)$ & $\mathrm{C}(22)-\mathrm{H}(22 \mathrm{~B})$ & 0.9800 \\
\hline $\mathrm{C}(10)-\mathrm{N}(3)$ & $1.52(3)$ & $\mathrm{C}(22)-\mathrm{H}(22 \mathrm{C})$ & 0.9800 \\
\hline$C(11)-C(12)$ & $1.41(3)$ & $\mathrm{C}(23)-\mathrm{H}(23 \mathrm{~A})$ & 0.9800 \\
\hline $\mathrm{C}(11)-\mathrm{H}(11)$ & 0.9500 & $\mathrm{C}(23)-\mathrm{H}(23 \mathrm{~B})$ & 0.9800 \\
\hline $\mathrm{C}(12)-\mathrm{N}(4)$ & $1.38(3)$ & $\mathrm{C}(23)-\mathrm{H}(23 \mathrm{C})$ & 0.9800 \\
\hline $\mathrm{C}(12)-\mathrm{C}(13)$ & $1.42(3)$ & $C(24)-C(25)$ & $1.38(3)$ \\
\hline$C(13)-F(2)$ & $1.25(3)$ & $C(24)-C(31)$ & $1.45(4)$ \\
\hline$C(13)-C(14)$ & $1.39(3)$ & $\mathrm{C}(24)-\mathrm{N}(8)$ & $1.49(3)$ \\
\hline$C(14)-C(15)$ & $1.42(3)$ & $\mathrm{C}(25)-\mathrm{F}(3)$ & $1.32(3)$ \\
\hline
\end{tabular}




\begin{tabular}{|c|c|c|c|}
\hline$C(25)-C(26)$ & $1.46(3)$ & $\mathrm{C}(39)-\mathrm{H}(39 \mathrm{~B})$ & 0.9800 \\
\hline$C(26)-C(29)$ & $1.42(3)$ & $\mathrm{C}(39)-\mathrm{H}(39 \mathrm{C})$ & 0.9800 \\
\hline$C(26)-C(27)$ & $1.43(3)$ & $\mathrm{C}(40)-\mathrm{O}(4)$ & $1.31(3)$ \\
\hline $\mathrm{C}(27)-\mathrm{O}(3)$ & $1.22(3)$ & $\mathrm{C}(40)-\mathrm{N}(10)$ & $1.32(4)$ \\
\hline$C(27)-N(6)$ & $1.38(3)$ & $\mathrm{C}(40)-\mathrm{N}(9)$ & $1.38(4)$ \\
\hline $\mathrm{C}(28)-\mathrm{N}(7)$ & $1.26(3)$ & $\mathrm{C}(41)-\mathrm{N}(10)$ & $1.42(4)$ \\
\hline $\mathrm{C}(28)-\mathrm{N}(6)$ & $1.41(3)$ & $\mathrm{C}(41)-\mathrm{C}(42)$ & $1.60(4)$ \\
\hline $\mathrm{C}(28)-\mathrm{H}(28)$ & 0.9500 & $\mathrm{C}(41)-\mathrm{H}(41 \mathrm{~A})$ & 0.9900 \\
\hline $\mathrm{C}(29)-\mathrm{N}(7)$ & $1.39(3)$ & $\mathrm{C}(41)-\mathrm{H}(41 \mathrm{~B})$ & 0.9900 \\
\hline$C(29)-C(30)$ & $1.42(3)$ & $\mathrm{C}(42)-\mathrm{C}(43)$ & $1.53(4)$ \\
\hline$C(30)-C(31)$ & $1.28(4)$ & $\mathrm{C}(42)-\mathrm{H}(42 \mathrm{~A})$ & 0.9900 \\
\hline $\mathrm{C}(30)-\mathrm{H}(30)$ & 0.9500 & $\mathrm{C}(42)-\mathrm{H}(42 \mathrm{~B})$ & 0.9900 \\
\hline $\mathrm{C}(31)-\mathrm{H}(31)$ & 0.9500 & $\mathrm{C}(43)-\mathrm{C}(46)$ & $1.40(4)$ \\
\hline$C(32)-N(6)$ & $1.40(3)$ & $\mathrm{C}(43)-\mathrm{C}(45)$ & $1.45(4)$ \\
\hline $\mathrm{C}(32)-\mathrm{H}(32 \mathrm{~A})$ & 0.9800 & $\mathrm{C}(43)-\mathrm{C}(44)$ & $1.50(5)$ \\
\hline C(32)-H(32B) & 0.9800 & $\mathrm{C}(44)-\mathrm{H}(44 \mathrm{~A})$ & 0.9800 \\
\hline $\mathrm{C}(32)-\mathrm{H}(32 \mathrm{C})$ & 0.9800 & $\mathrm{C}(44)-\mathrm{H}(44 \mathrm{~B})$ & 0.9800 \\
\hline $\mathrm{C}(33)-\mathrm{N}(8)$ & $1.37(3)$ & $\mathrm{C}(44)-\mathrm{H}(44 \mathrm{C})$ & 0.9800 \\
\hline$C(33)-C(34)$ & $1.38(3)$ & $\mathrm{C}(45)-\mathrm{H}(45 \mathrm{~A})$ & 0.9800 \\
\hline$C(33)-C(38)$ & $1.40(3)$ & $\mathrm{C}(45)-\mathrm{H}(45 \mathrm{~B})$ & 0.9800 \\
\hline$C(34)-C(35)$ & $1.38(3)$ & $\mathrm{C}(45)-\mathrm{H}(45 \mathrm{C})$ & 0.9800 \\
\hline $\mathrm{C}(34)-\mathrm{H}(34)$ & 0.9500 & $\mathrm{C}(46)-\mathrm{H}(46 \mathrm{~A})$ & 0.9800 \\
\hline$C(35)-C(36)$ & $1.40(3)$ & $\mathrm{C}(46)-\mathrm{H}(46 \mathrm{~B})$ & 0.9800 \\
\hline $\mathrm{C}(35)-\mathrm{N}(9)$ & $1.40(3)$ & $\mathrm{C}(46)-\mathrm{H}(46 \mathrm{C})$ & 0.9800 \\
\hline$C(36)-F(4)$ & $1.33(2)$ & $\mathrm{N}(3)-\mathrm{H}(3)$ & 0.8800 \\
\hline$C(36)-C(37)$ & $1.44(3)$ & $\mathrm{N}(4)-\mathrm{H}(4)$ & 0.8800 \\
\hline C(37)-C(38) & $1.34(3)$ & $\mathrm{N}(5)-\mathrm{H}(5 \mathrm{~A})$ & 0.8800 \\
\hline $\mathrm{C}(37)-\mathrm{H}(37)$ & 0.9500 & $\mathrm{~N}(8)-\mathrm{H}(8 \mathrm{~A})$ & 0.8800 \\
\hline C(38)-C(39) & $1.51(3)$ & $\mathrm{N}(9)-\mathrm{H}(9)$ & 0.8800 \\
\hline C(39)-H(39A) & 0.9800 & $\mathrm{~N}(10)-\mathrm{H}(10)$ & 0.8800 \\
\hline $\mathrm{C}(8)-\mathrm{C}(1)-\mathrm{N}(3)$ & $126(2)$ & $\mathrm{C}(1)-\mathrm{C}(2)-\mathrm{F}(1)$ & $115(2)$ \\
\hline$C(8)-C(1)-C(2)$ & $116(2)$ & $\mathrm{C}(2)-\mathrm{C}(3)-\mathrm{C}(6)$ & $117(2)$ \\
\hline $\mathrm{N}(3)-\mathrm{C}(1)-\mathrm{C}(2)$ & $118(2)$ & $\mathrm{C}(2)-\mathrm{C}(3)-\mathrm{C}(4)$ & $123(2)$ \\
\hline$C(3)-C(2)-C(1)$ & $125(2)$ & $\mathrm{C}(6)-\mathrm{C}(3)-\mathrm{C}(4)$ & $120(2)$ \\
\hline$C(3)-C(2)-F(1)$ & 119.3(19) & $\mathrm{O}(1)-\mathrm{C}(4)-\mathrm{N}(1)$ & $122(2)$ \\
\hline
\end{tabular}




\begin{tabular}{|c|c|c|c|}
\hline $\mathrm{O}(1)-\mathrm{C}(4)-\mathrm{C}(3)$ & $128(2)$ & $C(10)-C(15)-C(16)$ & $126(2)$ \\
\hline $\mathrm{N}(1)-\mathrm{C}(4)-\mathrm{C}(3)$ & $109.3(19)$ & $C(14)-C(15)-C(16)$ & $116(2)$ \\
\hline $\mathrm{N}(2)-\mathrm{C}(5)-\mathrm{N}(1)$ & $123(2)$ & $C(15)-C(16)-H(16 A)$ & 109.5 \\
\hline $\mathrm{N}(2)-\mathrm{C}(5)-\mathrm{H}(5)$ & 118.5 & $\mathrm{C}(15)-\mathrm{C}(16)-\mathrm{H}(16 \mathrm{~B})$ & 109.5 \\
\hline $\mathrm{N}(1)-\mathrm{C}(5)-\mathrm{H}(5)$ & 118.5 & $\mathrm{H}(16 \mathrm{~A})-\mathrm{C}(16)-\mathrm{H}(16 \mathrm{~B})$ & 109.5 \\
\hline $\mathrm{C}(7)-\mathrm{C}(6)-\mathrm{N}(2)$ & $120.2(19)$ & $\mathrm{C}(15)-\mathrm{C}(16)-\mathrm{H}(16 \mathrm{C})$ & 109.5 \\
\hline$C(7)-C(6)-C(3)$ & $121(2)$ & $\mathrm{H}(16 \mathrm{~A})-\mathrm{C}(16)-\mathrm{H}(16 \mathrm{C})$ & 109.5 \\
\hline $\mathrm{N}(2)-\mathrm{C}(6)-\mathrm{C}(3)$ & $119.2(18)$ & $\mathrm{H}(16 \mathrm{~B})-\mathrm{C}(16)-\mathrm{H}(16 \mathrm{C})$ & 109.5 \\
\hline$C(6)-C(7)-C(8)$ & $118(2)$ & $\mathrm{O}(2)-\mathrm{C}(17)-\mathrm{N}(5)$ & $121(3)$ \\
\hline $\mathrm{C}(6)-\mathrm{C}(7)-\mathrm{H}(7)$ & 121.0 & $\mathrm{O}(2)-\mathrm{C}(17)-\mathrm{N}(4)$ & $123(3)$ \\
\hline $\mathrm{C}(8)-\mathrm{C}(7)-\mathrm{H}(7)$ & 121.0 & $\mathrm{~N}(5)-\mathrm{C}(17)-\mathrm{N}(4)$ & $116(3)$ \\
\hline $\mathrm{C}(1)-\mathrm{C}(8)-\mathrm{C}(7)$ & $123(2)$ & $\mathrm{C}(19)-\mathrm{C}(18)-\mathrm{N}(5)$ & $115(3)$ \\
\hline $\mathrm{C}(1)-\mathrm{C}(8)-\mathrm{H}(8)$ & 118.5 & $\mathrm{C}(19)-\mathrm{C}(18)-\mathrm{H}(18 \mathrm{~A})$ & 108.5 \\
\hline $\mathrm{C}(7)-\mathrm{C}(8)-\mathrm{H}(8)$ & 118.5 & $\mathrm{~N}(5)-\mathrm{C}(18)-\mathrm{H}(18 \mathrm{~A})$ & 108.5 \\
\hline $\mathrm{N}(1)-\mathrm{C}(9)-\mathrm{H}(9 \mathrm{~A})$ & 109.5 & $\mathrm{C}(19)-\mathrm{C}(18)-\mathrm{H}(18 \mathrm{~B})$ & 108.5 \\
\hline $\mathrm{N}(1)-\mathrm{C}(9)-\mathrm{H}(9 \mathrm{~B})$ & 109.5 & $\mathrm{~N}(5)-\mathrm{C}(18)-\mathrm{H}(18 \mathrm{~B})$ & 108.5 \\
\hline $\mathrm{H}(9 \mathrm{~A})-\mathrm{C}(9)-\mathrm{H}(9 \mathrm{~B})$ & 109.5 & $\mathrm{H}(18 \mathrm{~A})-\mathrm{C}(18)-\mathrm{H}(18 \mathrm{~B})$ & 107.5 \\
\hline $\mathrm{N}(1)-\mathrm{C}(9)-\mathrm{H}(9 \mathrm{C})$ & 109.5 & $C(18)-C(19)-C(20)$ & $123(3)$ \\
\hline $\mathrm{H}(9 \mathrm{~A})-\mathrm{C}(9)-\mathrm{H}(9 \mathrm{C})$ & 109.5 & $\mathrm{C}(18)-\mathrm{C}(19)-\mathrm{H}(19 \mathrm{~A})$ & 106.5 \\
\hline $\mathrm{H}(9 \mathrm{~B})-\mathrm{C}(9)-\mathrm{H}(9 \mathrm{C})$ & 109.5 & $\mathrm{C}(20)-\mathrm{C}(19)-\mathrm{H}(19 \mathrm{~A})$ & 106.5 \\
\hline$C(15)-C(10)-C(11)$ & $126(2)$ & $\mathrm{C}(18)-\mathrm{C}(19)-\mathrm{H}(19 \mathrm{~B})$ & 106.5 \\
\hline $\mathrm{C}(15)-\mathrm{C}(10)-\mathrm{N}(3)$ & $120(2)$ & $\mathrm{C}(20)-\mathrm{C}(19)-\mathrm{H}(19 \mathrm{~B})$ & 106.5 \\
\hline $\mathrm{C}(11)-\mathrm{C}(10)-\mathrm{N}(3)$ & $114(2)$ & $\mathrm{H}(19 \mathrm{~A})-\mathrm{C}(19)-\mathrm{H}(19 \mathrm{~B})$ & 106.5 \\
\hline$C(10)-C(11)-C(12)$ & $119(2)$ & $C(21)-C(20)-C(19)$ & $118(3)$ \\
\hline $\mathrm{C}(10)-\mathrm{C}(11)-\mathrm{H}(11)$ & 120.4 & $C(21)-C(20)-C(22)$ & $106(3)$ \\
\hline $\mathrm{C}(12)-\mathrm{C}(11)-\mathrm{H}(11)$ & 120.4 & $C(19)-C(20)-C(22)$ & $108(2)$ \\
\hline $\mathrm{N}(4)-\mathrm{C}(12)-\mathrm{C}(11)$ & $121(2)$ & $\mathrm{C}(21)-\mathrm{C}(20)-\mathrm{C}(23)$ & 113(3) \\
\hline $\mathrm{N}(4)-\mathrm{C}(12)-\mathrm{C}(13)$ & $122.5(19)$ & $C(19)-C(20)-C(23)$ & $110(2)$ \\
\hline$C(11)-C(12)-C(13)$ & $117(2)$ & $C(22)-C(20)-C(23)$ & $101(2)$ \\
\hline $\mathrm{F}(2)-\mathrm{C}(13)-\mathrm{C}(14)$ & $120(2)$ & $\mathrm{C}(20)-\mathrm{C}(21)-\mathrm{H}(21 \mathrm{~A})$ & 109.5 \\
\hline $\mathrm{F}(2)-\mathrm{C}(13)-\mathrm{C}(12)$ & $120(2)$ & $\mathrm{C}(20)-\mathrm{C}(21)-\mathrm{H}(21 \mathrm{~B})$ & 109.5 \\
\hline$C(14)-C(13)-C(12)$ & $120(2)$ & $\mathrm{H}(21 \mathrm{~A})-\mathrm{C}(21)-\mathrm{H}(21 \mathrm{~B})$ & 109.5 \\
\hline$C(13)-C(14)-C(15)$ & $120(2)$ & $\mathrm{C}(20)-\mathrm{C}(21)-\mathrm{H}(21 \mathrm{C})$ & 109.5 \\
\hline $\mathrm{C}(13)-\mathrm{C}(14)-\mathrm{H}(14)$ & 120.0 & $\mathrm{H}(21 \mathrm{~A})-\mathrm{C}(21)-\mathrm{H}(21 \mathrm{C})$ & 109.5 \\
\hline $\mathrm{C}(15)-\mathrm{C}(14)-\mathrm{H}(14)$ & 120.0 & $\mathrm{H}(21 \mathrm{~B})-\mathrm{C}(21)-\mathrm{H}(21 \mathrm{C})$ & 109.5 \\
\hline$C(10)-C(15)-C(14)$ & $118(2)$ & $\mathrm{C}(20)-\mathrm{C}(22)-\mathrm{H}(22 \mathrm{~A})$ & 109.5 \\
\hline
\end{tabular}




\begin{tabular}{|c|c|c|c|}
\hline $\mathrm{C}(20)-\mathrm{C}(22)-\mathrm{H}(22 \mathrm{~B})$ & 109.5 & $\mathrm{~N}(6)-\mathrm{C}(32)-\mathrm{H}(32 \mathrm{~B})$ & 109.5 \\
\hline $\mathrm{H}(22 \mathrm{~A})-\mathrm{C}(22)-\mathrm{H}(22 \mathrm{~B})$ & 109.5 & $\mathrm{H}(32 \mathrm{~A})-\mathrm{C}(32)-\mathrm{H}(32 \mathrm{~B})$ & 109.5 \\
\hline $\mathrm{C}(20)-\mathrm{C}(22)-\mathrm{H}(22 \mathrm{C})$ & 109.5 & $\mathrm{~N}(6)-\mathrm{C}(32)-\mathrm{H}(32 \mathrm{C})$ & 109.5 \\
\hline $\mathrm{H}(22 \mathrm{~A})-\mathrm{C}(22)-\mathrm{H}(22 \mathrm{C})$ & 109.5 & $\mathrm{H}(32 \mathrm{~A})-\mathrm{C}(32)-\mathrm{H}(32 \mathrm{C})$ & 109.5 \\
\hline $\mathrm{H}(22 \mathrm{~B})-\mathrm{C}(22)-\mathrm{H}(22 \mathrm{C})$ & 109.5 & H(32B)-C(32)-H(32C) & 109.5 \\
\hline $\mathrm{C}(20)-\mathrm{C}(23)-\mathrm{H}(23 \mathrm{~A})$ & 109.5 & $\mathrm{~N}(8)-\mathrm{C}(33)-\mathrm{C}(34)$ & $122(2)$ \\
\hline $\mathrm{C}(20)-\mathrm{C}(23)-\mathrm{H}(23 \mathrm{~B})$ & 109.5 & $\mathrm{~N}(8)-\mathrm{C}(33)-\mathrm{C}(38)$ & $116(2)$ \\
\hline $\mathrm{H}(23 \mathrm{~A})-\mathrm{C}(23)-\mathrm{H}(23 \mathrm{~B})$ & 109.5 & $C(34)-C(33)-C(38)$ & $121(2)$ \\
\hline $\mathrm{C}(20)-\mathrm{C}(23)-\mathrm{H}(23 \mathrm{C})$ & 109.5 & $\mathrm{C}(33)-\mathrm{C}(34)-\mathrm{C}(35)$ & $121(2)$ \\
\hline $\mathrm{H}(23 \mathrm{~A})-\mathrm{C}(23)-\mathrm{H}(23 \mathrm{C})$ & 109.5 & $\mathrm{C}(33)-\mathrm{C}(34)-\mathrm{H}(34)$ & 119.3 \\
\hline $\mathrm{H}(23 \mathrm{~B})-\mathrm{C}(23)-\mathrm{H}(23 \mathrm{C})$ & 109.5 & $\mathrm{C}(35)-\mathrm{C}(34)-\mathrm{H}(34)$ & 119.3 \\
\hline$C(25)-C(24)-C(31)$ & $117(2)$ & $C(34)-C(35)-C(36)$ & $118(2)$ \\
\hline $\mathrm{C}(25)-\mathrm{C}(24)-\mathrm{N}(8)$ & $117(2)$ & $\mathrm{C}(34)-\mathrm{C}(35)-\mathrm{N}(9)$ & $124(2)$ \\
\hline $\mathrm{C}(31)-\mathrm{C}(24)-\mathrm{N}(8)$ & $126(2)$ & $\mathrm{C}(36)-\mathrm{C}(35)-\mathrm{N}(9)$ & $118(2)$ \\
\hline $\mathrm{F}(3)-\mathrm{C}(25)-\mathrm{C}(24)$ & $119(2)$ & $\mathrm{F}(4)-\mathrm{C}(36)-\mathrm{C}(35)$ & $121.6(19)$ \\
\hline$F(3)-C(25)-C(26)$ & $121.8(18)$ & $\mathrm{F}(4)-\mathrm{C}(36)-\mathrm{C}(37)$ & $118.8(19)$ \\
\hline $\mathrm{C}(24)-\mathrm{C}(25)-\mathrm{C}(26)$ & $119(2)$ & $C(35)-C(36)-C(37)$ & $118.8(19)$ \\
\hline $\mathrm{C}(29)-\mathrm{C}(26)-\mathrm{C}(27)$ & $119(2)$ & $\mathrm{C}(38)-\mathrm{C}(37)-\mathrm{C}(36)$ & $122(2)$ \\
\hline$C(29)-C(26)-C(25)$ & $120.5(19)$ & $\mathrm{C}(38)-\mathrm{C}(37)-\mathrm{H}(37)$ & 118.9 \\
\hline$C(27)-C(26)-C(25)$ & $120(2)$ & $\mathrm{C}(36)-\mathrm{C}(37)-\mathrm{H}(37)$ & 118.9 \\
\hline $\mathrm{O}(3)-\mathrm{C}(27)-\mathrm{N}(6)$ & $117(2)$ & $\mathrm{C}(37)-\mathrm{C}(38)-\mathrm{C}(33)$ & $118(2)$ \\
\hline $\mathrm{O}(3)-\mathrm{C}(27)-\mathrm{C}(26)$ & $128(2)$ & $\mathrm{C}(37)-\mathrm{C}(38)-\mathrm{C}(39)$ & $121(2)$ \\
\hline $\mathrm{N}(6)-\mathrm{C}(27)-\mathrm{C}(26)$ & $115(2)$ & $\mathrm{C}(33)-\mathrm{C}(38)-\mathrm{C}(39)$ & $121(2)$ \\
\hline $\mathrm{N}(7)-\mathrm{C}(28)-\mathrm{N}(6)$ & $125(2)$ & $\mathrm{C}(38)-\mathrm{C}(39)-\mathrm{H}(39 \mathrm{~A})$ & 109.5 \\
\hline $\mathrm{N}(7)-\mathrm{C}(28)-\mathrm{H}(28)$ & 117.7 & $\mathrm{C}(38)-\mathrm{C}(39)-\mathrm{H}(39 \mathrm{~B})$ & 109.5 \\
\hline $\mathrm{N}(6)-\mathrm{C}(28)-\mathrm{H}(28)$ & 117.7 & H(39A)-C(39)-H(39B) & 109.5 \\
\hline$N(7)-C(29)-C(30)$ & $122(2)$ & $\mathrm{C}(38)-\mathrm{C}(39)-\mathrm{H}(39 \mathrm{C})$ & 109.5 \\
\hline$N(7)-C(29)-C(26)$ & $121.7(18)$ & H(39A)-C(39)-H(39C) & 109.5 \\
\hline$C(30)-C(29)-C(26)$ & $116(2)$ & H(39B)-C(39)-H(39C) & 109.5 \\
\hline $\mathrm{C}(31)-\mathrm{C}(30)-\mathrm{C}(29)$ & $123(3)$ & $\mathrm{O}(4)-\mathrm{C}(40)-\mathrm{N}(10)$ & $127(3)$ \\
\hline $\mathrm{C}(31)-\mathrm{C}(30)-\mathrm{H}(30)$ & 118.4 & $\mathrm{O}(4)-\mathrm{C}(40)-\mathrm{N}(9)$ & $117(3)$ \\
\hline $\mathrm{C}(29)-\mathrm{C}(30)-\mathrm{H}(30)$ & 118.4 & $\mathrm{~N}(10)-\mathrm{C}(40)-\mathrm{N}(9)$ & $116(3)$ \\
\hline$C(30)-C(31)-C(24)$ & $124(3)$ & $\mathrm{N}(10)-\mathrm{C}(41)-\mathrm{C}(42)$ & $111(2)$ \\
\hline $\mathrm{C}(30)-\mathrm{C}(31)-\mathrm{H}(31)$ & 118.2 & $\mathrm{~N}(10)-\mathrm{C}(41)-\mathrm{H}(41 \mathrm{~A})$ & 109.4 \\
\hline $\mathrm{C}(24)-\mathrm{C}(31)-\mathrm{H}(31)$ & 118.2 & $\mathrm{C}(42)-\mathrm{C}(41)-\mathrm{H}(41 \mathrm{~A})$ & 109.4 \\
\hline $\mathrm{N}(6)-\mathrm{C}(32)-\mathrm{H}(32 \mathrm{~A})$ & 109.5 & $\mathrm{~N}(10)-\mathrm{C}(41)-\mathrm{H}(41 \mathrm{~B})$ & 109.4 \\
\hline
\end{tabular}




\begin{tabular}{|c|c|c|c|}
\hline $\mathrm{C}(42)-\mathrm{C}(41)-\mathrm{H}(41 \mathrm{~B})$ & 109.4 & $\mathrm{C}(1)-\mathrm{N}(3)-\mathrm{C}(10)$ & $125.6(19)$ \\
\hline $\mathrm{H}(41 \mathrm{~A})-\mathrm{C}(41)-\mathrm{H}(41 \mathrm{~B})$ & 108.0 & $\mathrm{C}(1)-\mathrm{N}(3)-\mathrm{H}(3)$ & 117.2 \\
\hline$C(43)-C(42)-C(41)$ & $117(2)$ & $\mathrm{C}(10)-\mathrm{N}(3)-\mathrm{H}(3)$ & 117.2 \\
\hline $\mathrm{C}(43)-\mathrm{C}(42)-\mathrm{H}(42 \mathrm{~A})$ & 108.1 & $\mathrm{C}(17)-\mathrm{N}(4)-\mathrm{C}(12)$ & $118(2)$ \\
\hline $\mathrm{C}(41)-\mathrm{C}(42)-\mathrm{H}(42 \mathrm{~A})$ & 108.1 & $\mathrm{C}(17)-\mathrm{N}(4)-\mathrm{H}(4)$ & 121.1 \\
\hline $\mathrm{C}(43)-\mathrm{C}(42)-\mathrm{H}(42 \mathrm{~B})$ & 108.1 & $\mathrm{C}(12)-\mathrm{N}(4)-\mathrm{H}(4)$ & 121.1 \\
\hline $\mathrm{C}(41)-\mathrm{C}(42)-\mathrm{H}(42 \mathrm{~B})$ & 108.1 & $\mathrm{C}(17)-\mathrm{N}(5)-\mathrm{C}(18)$ & $120(3)$ \\
\hline $\mathrm{H}(42 \mathrm{~A})-\mathrm{C}(42)-\mathrm{H}(42 \mathrm{~B})$ & 107.3 & $\mathrm{C}(17)-\mathrm{N}(5)-\mathrm{H}(5 \mathrm{~A})$ & 120.2 \\
\hline$C(46)-C(43)-C(45)$ & $107(3)$ & $\mathrm{C}(18)-\mathrm{N}(5)-\mathrm{H}(5 \mathrm{~A})$ & 120.2 \\
\hline$C(46)-C(43)-C(44)$ & $113(3)$ & $C(27)-N(6)-C(32)$ & $119.6(19)$ \\
\hline$C(45)-C(43)-C(44)$ & $107(3)$ & $\mathrm{C}(27)-\mathrm{N}(6)-\mathrm{C}(28)$ & $121.2(19)$ \\
\hline$C(46)-C(43)-C(42)$ & $113(3)$ & $\mathrm{C}(32)-\mathrm{N}(6)-\mathrm{C}(28)$ & $119(2)$ \\
\hline$C(45)-C(43)-C(42)$ & $101(3)$ & $\mathrm{C}(28)-\mathrm{N}(7)-\mathrm{C}(29)$ & $117.9(19)$ \\
\hline$C(44)-C(43)-C(42)$ & $115(3)$ & $\mathrm{C}(33)-\mathrm{N}(8)-\mathrm{C}(24)$ & $122(2)$ \\
\hline $\mathrm{C}(43)-\mathrm{C}(44)-\mathrm{H}(44 \mathrm{~A})$ & 109.5 & $\mathrm{C}(33)-\mathrm{N}(8)-\mathrm{H}(8 \mathrm{~A})$ & 118.8 \\
\hline $\mathrm{C}(43)-\mathrm{C}(44)-\mathrm{H}(44 \mathrm{~B})$ & 109.5 & $\mathrm{C}(24)-\mathrm{N}(8)-\mathrm{H}(8 \mathrm{~A})$ & 118.8 \\
\hline $\mathrm{H}(44 \mathrm{~A})-\mathrm{C}(44)-\mathrm{H}(44 \mathrm{~B})$ & 109.5 & $\mathrm{C}(40)-\mathrm{N}(9)-\mathrm{C}(35)$ & $129(2)$ \\
\hline $\mathrm{C}(43)-\mathrm{C}(44)-\mathrm{H}(44 \mathrm{C})$ & 109.5 & $\mathrm{C}(40)-\mathrm{N}(9)-\mathrm{H}(9)$ & 115.3 \\
\hline $\mathrm{H}(44 \mathrm{~A})-\mathrm{C}(44)-\mathrm{H}(44 \mathrm{C})$ & 109.5 & $\mathrm{C}(35)-\mathrm{N}(9)-\mathrm{H}(9)$ & 115.3 \\
\hline $\mathrm{H}(44 \mathrm{~B})-\mathrm{C}(44)-\mathrm{H}(44 \mathrm{C})$ & 109.5 & $\mathrm{C}(40)-\mathrm{N}(10)-\mathrm{C}(41)$ & $123(3)$ \\
\hline $\mathrm{C}(43)-\mathrm{C}(45)-\mathrm{H}(45 \mathrm{~A})$ & 109.5 & $\mathrm{C}(40)-\mathrm{N}(10)-\mathrm{H}(10)$ & 118.5 \\
\hline $\mathrm{C}(43)-\mathrm{C}(45)-\mathrm{H}(45 \mathrm{~B})$ & 109.5 & $\mathrm{C}(41)-\mathrm{N}(10)-\mathrm{H}(10)$ & 118.5 \\
\hline $\mathrm{H}(45 \mathrm{~A})-\mathrm{C}(45)-\mathrm{H}(45 \mathrm{~B})$ & 109.5 & & \\
\hline $\mathrm{C}(43)-\mathrm{C}(45)-\mathrm{H}(45 \mathrm{C})$ & 109.5 & & \\
\hline $\mathrm{H}(45 \mathrm{~A})-\mathrm{C}(45)-\mathrm{H}(45 \mathrm{C})$ & 109.5 & & \\
\hline $\mathrm{H}(45 \mathrm{~B})-\mathrm{C}(45)-\mathrm{H}(45 \mathrm{C})$ & 109.5 & & \\
\hline $\mathrm{C}(43)-\mathrm{C}(46)-\mathrm{H}(46 \mathrm{~A})$ & 109.5 & & \\
\hline $\mathrm{C}(43)-\mathrm{C}(46)-\mathrm{H}(46 \mathrm{~B})$ & 109.5 & & \\
\hline $\mathrm{H}(46 \mathrm{~A})-\mathrm{C}(46)-\mathrm{H}(46 \mathrm{~B})$ & 109.5 & & \\
\hline$C(43)-C(46)-H(46 C)$ & 109.5 & & \\
\hline $\mathrm{H}(46 \mathrm{~A})-\mathrm{C}(46)-\mathrm{H}(46 \mathrm{C})$ & 109.5 & & \\
\hline $\mathrm{H}(46 \mathrm{~B})-\mathrm{C}(46)-\mathrm{H}(46 \mathrm{C})$ & 109.5 & & \\
\hline $\mathrm{C}(5)-\mathrm{N}(1)-\mathrm{C}(4)$ & $126.5(19)$ & & \\
\hline $\mathrm{C}(5)-\mathrm{N}(1)-\mathrm{C}(9)$ & $120.4(19)$ & & \\
\hline $\mathrm{C}(4)-\mathrm{N}(1)-\mathrm{C}(9)$ & $113.0(17)$ & & \\
\hline$C(5)-N(2)-C(6)$ & $120.8(19)$ & & \\
\hline
\end{tabular}


Symmetry transformations used to generate equivalent atoms:

Hydrogen coordinates $\left(\mathrm{x} 10^{4}\right)$ and isotropic displacement parameters $\left(\AA^{2} \times 10^{3}\right)$.

\begin{tabular}{|c|c|c|c|c|}
\hline & $\mathrm{x}$ & $\mathrm{y}$ & $\mathrm{z}$ & $\mathrm{U}(\mathrm{eq})$ \\
\hline $\mathrm{H}(5)$ & 5257 & 8357 & 3745 & 23 \\
\hline $\mathrm{H}(7)$ & 4194 & 5933 & 3930 & 26 \\
\hline $\mathrm{H}(8)$ & 4045 & 3379 & 4293 & 28 \\
\hline $\mathrm{H}(9 \mathrm{~A})$ & 5861 & 7798 & 3736 & 33 \\
\hline $\mathrm{H}(9 \mathrm{~B})$ & 5921 & 6281 & 3121 & 33 \\
\hline $\mathrm{H}(9 \mathrm{C})$ & 5991 & 6227 & 4287 & 33 \\
\hline $\mathrm{H}(11)$ & 4067 & 1451 & 5450 & 28 \\
\hline $\mathrm{H}(14)$ & 3401 & -2067 & 3354 & 24 \\
\hline $\mathrm{H}(16 \mathrm{~A})$ & 4021 & -457 & 2243 & 38 \\
\hline $\mathrm{H}(16 \mathrm{~B})$ & 3842 & -2112 & 2387 & 38 \\
\hline $\mathrm{H}(16 \mathrm{C})$ & 4261 & -1762 & 2860 & 38 \\
\hline $\mathrm{H}(18 \mathrm{~A})$ & 3010 & -1584 & 8247 & 76 \\
\hline $\mathrm{H}(18 \mathrm{~B})$ & 3446 & -1487 & 8601 & 76 \\
\hline $\mathrm{H}(19 \mathrm{~A})$ & 3003 & 770 & 9068 & 82 \\
\hline $\mathrm{H}(19 \mathrm{~B})$ & 3398 & 236 & 9594 & 82 \\
\hline $\mathrm{H}(21 \mathrm{~A})$ & 2449 & -534 & 9588 & 123 \\
\hline $\mathrm{H}(21 \mathrm{~B})$ & 2517 & -1845 & 10407 & 123 \\
\hline $\mathrm{H}(21 \mathrm{C})$ & 2588 & -2207 & 9317 & 123 \\
\hline $\mathrm{H}(22 \mathrm{~A})$ & 3056 & -3024 & 10796 & 74 \\
\hline $\mathrm{H}(22 \mathrm{~B})$ & 3433 & -2089 & 10777 & 74 \\
\hline $\mathrm{H}(22 \mathrm{C})$ & 3243 & -2976 & 9821 & 74 \\
\hline $\mathrm{H}(23 \mathrm{~A})$ & 2952 & 1150 & 10929 & 86 \\
\hline $\mathrm{H}(23 \mathrm{~B})$ & 3308 & 128 & 11316 & 86 \\
\hline $\mathrm{H}(23 \mathrm{C})$ & 2909 & -366 & 11561 & 86 \\
\hline $\mathrm{H}(28)$ & 5261 & 13319 & 11450 & 38 \\
\hline $\mathrm{H}(30)$ & 4213 & 10805 & 10295 & 36 \\
\hline $\mathrm{H}(31)$ & 4035 & 8411 & 10108 & 50 \\
\hline $\mathrm{H}(32 \mathrm{~A})$ & 5910 & 11289 & 12622 & 52 \\
\hline $\mathrm{H}(32 \mathrm{~B})$ & 5845 & 12720 & 11889 & 52 \\
\hline $\mathrm{H}(32 \mathrm{C})$ & 5985 & 11106 & 11518 & 52 \\
\hline $\mathrm{H}(34)$ & 4092 & 6291 & 8754 & 36 \\
\hline
\end{tabular}




\begin{tabular}{|c|c|c|c|c|}
\hline $\mathrm{H}(37)$ & 3415 & 3047 & 10494 & 26 \\
\hline $\mathrm{H}(39 \mathrm{~A})$ & 3852 & 3112 & 11871 & 38 \\
\hline $\mathrm{H}(39 \mathrm{~B})$ & 4073 & 4711 & 12006 & 38 \\
\hline $\mathrm{H}(39 \mathrm{C})$ & 4269 & 3216 & 11636 & 38 \\
\hline $\mathrm{H}(41 \mathrm{~A})$ & 2701 & 5255 & 5164 & 61 \\
\hline $\mathrm{H}(41 \mathrm{~B})$ & 2874 & 3618 & 5519 & 61 \\
\hline $\mathrm{H}(42 \mathrm{~A})$ & 3228 & 5686 & 4277 & 65 \\
\hline $\mathrm{H}(42 \mathrm{~B})$ & 3381 & 4013 & 4596 & 65 \\
\hline $\mathrm{H}(44 \mathrm{~A})$ & 2684 & 1909 & 3071 & 125 \\
\hline $\mathrm{H}(44 \mathrm{~B})$ & 2438 & 3038 & 3626 & 125 \\
\hline $\mathrm{H}(44 \mathrm{C})$ & 2776 & 2116 & 4228 & 125 \\
\hline $\mathrm{H}(45 \mathrm{~A})$ & 2812 & 5722 & 2565 & 91 \\
\hline $\mathrm{H}(45 \mathrm{~B})$ & 2709 & 6001 & 3636 & 91 \\
\hline $\mathrm{H}(45 \mathrm{C})$ & 2459 & 4882 & 2894 & 91 \\
\hline $\mathrm{H}(46 \mathrm{~A})$ & 3026 & 3672 & 2119 & 131 \\
\hline $\mathrm{H}(46 \mathrm{~B})$ & 3284 & 2615 & 2885 & 131 \\
\hline $\mathrm{H}(46 \mathrm{C})$ & 3370 & 4417 & 2812 & 131 \\
\hline $\mathrm{H}(3)$ & 4602 & 171 & 4005 & 36 \\
\hline $\mathrm{H}(4)$ & 3407 & 1666 & 6180 & 27 \\
\hline $\mathrm{H}(5 \mathrm{~A})$ & 3199 & 1186 & 7620 & 70 \\
\hline $\mathrm{H}(8 \mathrm{~A})$ & 4595 & 4947 & 10706 & 45 \\
\hline $\mathrm{H}(9)$ & 3457 & 6558 & 7586 & 24 \\
\hline $\mathrm{H}(10)$ & 3202 & 6395 & 6103 & 69 \\
\hline
\end{tabular}

Qualitative comparison of the intermolecular interaction networks in the small-molecule crystal structures of compounds 6 and 7

Each molecule of 6 (Figure S1) makes four classical hydrogen bonds and two non-classical $\mathrm{CH}-\mathrm{O}=\mathrm{C}$ interactions. In addition, the quinazolinone forms a $\pi$-stacking interaction with the quinazolinone ring of a second molecule. The tert-butyl group is located in a hydrophobic pocket build towards a quinazolinone from another molecule. In 7 (Figure S2), the guanidine group forms a head-to-tail chain with four hydrogen bonds with close to ideal orientation. However, the conjugation between the guanidine nitrogen and the fluoro-phenyl ring is weakened as the guanidine is rotated $58^{\circ}$ out of plane. The quinazolinone ring forms a stacking interaction to a quinazolinone from a second molecule as found for $\mathbf{6}$. The hydrophobic tert-butyl group in $\mathbf{7}$ is placed adjacent to a polar guanidine group. We conclude that the likely weaker hydrogen bonds in 6 compared to 7 are more than compensated by the stronger hydrophobic interactions and maintained conjugation on the urea substitution, which is compromised in compound 6. 
Table S5. Crystallography statistics for Figure 4 (X-ray crystal structure of GNE-0749 (7) bound in a mutant BRAF construct).

\begin{tabular}{|c|c|}
\hline & bRaf + GNE-0749 \\
\hline PDB code & $7 \mathrm{~K} 0 \mathrm{~V}$ \\
\hline Space group & $\mathrm{P} 2{ }_{1} 2_{1} 2_{1}$ \\
\hline Unit cell & $\begin{array}{c}a=87.2 \AA, b=115.4 \AA, c=119.4 \AA, \\
\alpha=\beta=\gamma=90^{\circ}\end{array}$ \\
\hline Resolution & $1.93 \AA$ \\
\hline Total measured reflections & $60440(3023)^{1}$ \\
\hline Completeness (\% spherical) & $66.7(11.0)$ \\
\hline Completeness ( $\%$ ellipsoidal) & $94.2(62.1)$ \\
\hline Redundancy & $6.6(6.6)$ \\
\hline $\mathrm{I} / \sigma$ & $11.4(1.8)$ \\
\hline Rsym $^{2}$ & $0.146(0.464)$ \\
\hline $\mathrm{CC}_{1 / 2}$ & $0.998(0.675)$ \\
\hline Resolution range & $50-1.93 \AA$ \\
\hline Rcryst $^{3} /$ Rfree $^{4}$ & $0.184 / 0.230$ \\
\hline Non-hydrogen atoms & 9803 \\
\hline Water molecules & 434 \\
\hline Average B & $37.3 \AA$ \\
\hline r.m.s.d. bond lengths & $0.007 \AA$ \\
\hline r.m.s.d. angles & $0.85^{\circ}$ \\
\hline Ramachandran $^{5}$ & $0.897 / 0.10 / 0.001 / 0.002$ \\
\hline
\end{tabular}

${ }^{1}$ Values in parentheses are of the highest resolution shell

${ }^{2} \mathrm{Rsym}=\Sigma\left|\mathrm{I}_{\mathrm{hi}}-\mathrm{I}_{\mathrm{h}}\right| / \Sigma \mathrm{I}_{\mathrm{hi}}$, where $\mathrm{I}_{\mathrm{hi}}$ is the scaled intensity of the $i$ th symmetry-related observation of reflection $h$ and $\mathrm{I}_{\mathrm{h}}$ is the mean value.

${ }^{3}$ Rcryst $=\Sigma_{h}\left|F_{o h}-F_{c h}\right| / \Sigma_{h} F_{o h}$, where $F_{o h}$ and $F_{c h}$ are the observed and calculated structure factor amplitudes for reflection $h$.

${ }^{4}$ Value of Rfree is calculated for 5\% randomly chosen reflections not included in the refinement.

${ }^{5}$ Values following Ramachandran indication fraction of residues fall into most favored/additional allowed/generally allowed/disallowed regions of Ramachandran plot. 

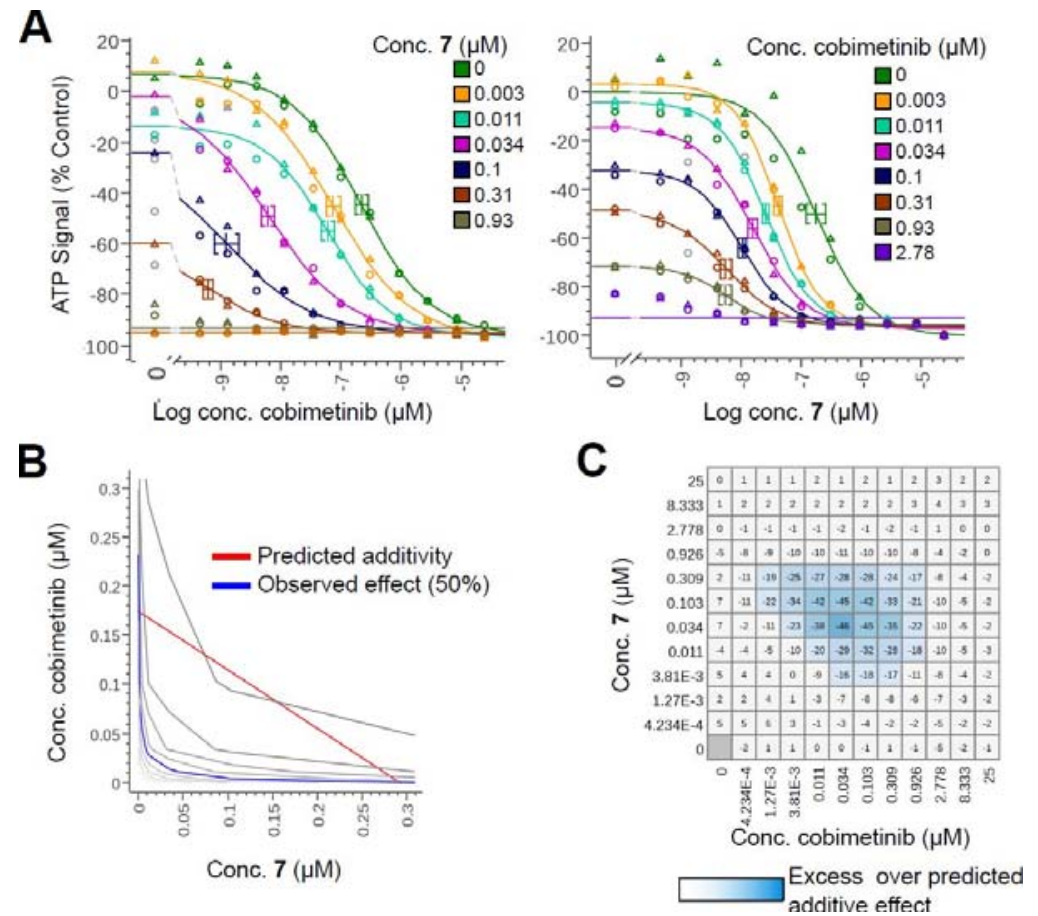

Figure S3. Synergistic effects of GNE-0749+cobimetinib in KRAS-mutant cell line, HCT116.

a) Proliferation of HCT116 cells, determined by incorporation of 5-ethynyl-2'-deoxyuridine (EdU) into newly synthesized DNA. b) Isobologram plot of fractional EdU-uptake inhibition at different dose combinations. c) Difference between observed percent effect and expected fractional effect according to the Loewe additivity model plotted for each dose combination.
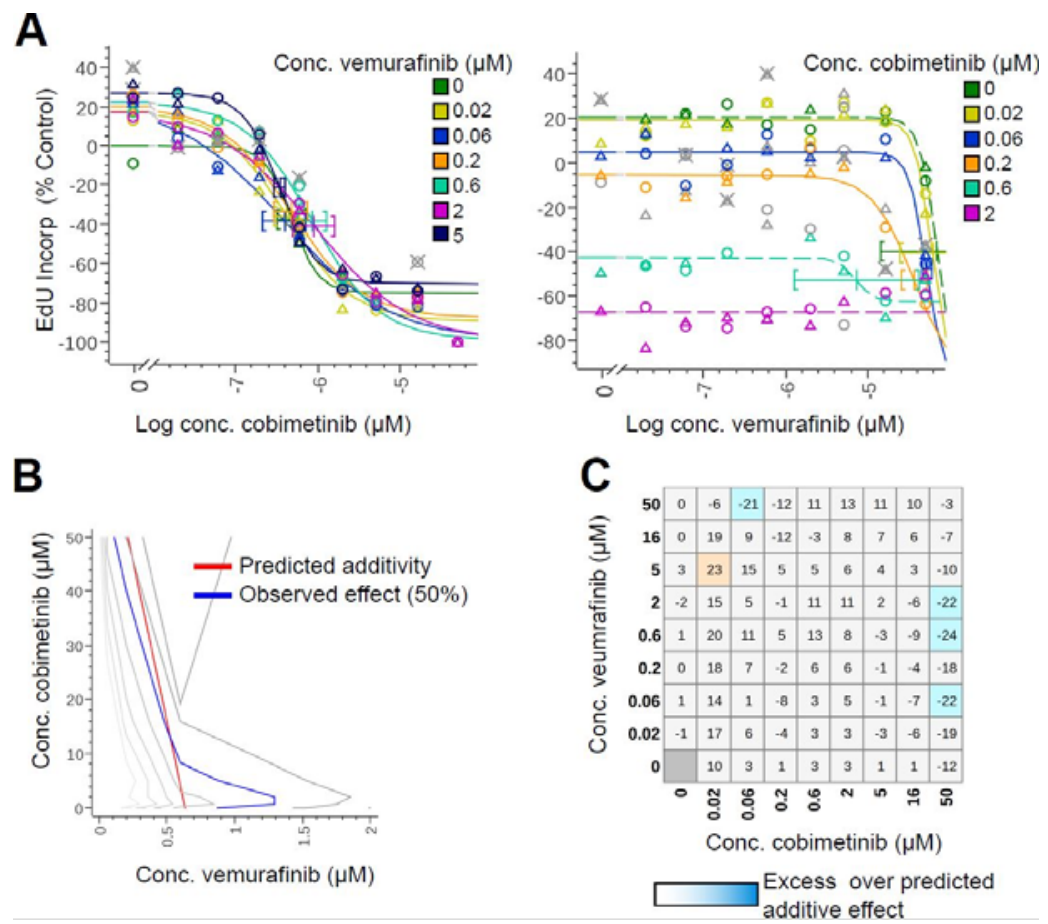

Figure S4. Non-synergistic effects of vemurafenib+cobimetinib in KRAS-mutant cell line, HCT116.

a) Proliferation of HCT116 cells, determined by incorporation of 5-ethynyl-2'-deoxyuridine (EdU) into newly synthesized DNA. b) Isobologram plot of fractional EdU-uptake inhibition at different dose combinations. c) Difference between observed percent effect and expected fractional effect according to the Loewe additivity model plotted for each dose combination. 
Scheme S1. Full synthetic details for compounds 2-7.

$$
\text { (2) }
$$

(a) $10 \% \mathrm{Pd} / \mathrm{C}\left(50 \% \mathrm{w} / \mathrm{w}\right.$ ), $\mathrm{H}_{2}(1 \mathrm{~atm}), \mathrm{EtOH}, 23^{\circ} \mathrm{C}, 94 \%$; (b) $\mathrm{PhOCOCl}\left(1.1\right.$ equiv), $\mathrm{NaHCO}_{3}$ (2 equiv), $\mathrm{THF}, 23^{\circ} \mathrm{C}-60^{\circ} \mathrm{C}$; then $\mathrm{NEt}_{3}$ (2 equiv), 3,3-dimethylbutylamine ( 1.1 equiv), toluene, $23^{\circ} \mathrm{C}-90^{\circ} \mathrm{C}, 20 \%$ ( 2 steps); (c) $\mathrm{LiOH} \cdot \mathrm{H}_{2} \mathrm{O}\left(1.1\right.$ equiv), $\mathrm{THF} / \mathrm{H}_{2} \mathrm{O}$ (5:1), $80^{\circ} \mathrm{C}, 97 \%$; (d) DMF (0.2 equiv), oxalyl chloride (3 equiv), THF, $23^{\circ} \mathrm{C}$; then 3-methoxy-1H-pyrazolo[3,4-b]pyridin-5-amine (1.2 equiv), $\mathrm{NEt}_{3}$ (2 equiv), $55^{\circ} \mathrm{C}, 48 \%$; (e) Hunig's base (2 equiv), $10 \%$ aq. $\mathrm{NaOH} / \mathrm{DMF}(2: 1), 23^{\circ} \mathrm{C}, 72 \%$.

$$
\text { (2steps) }
$$

(a) PhOCOCl (1.1 equiv), $\mathrm{NaHCO}_{3}$ (2 equiv), THF, $0{ }^{\circ} \mathrm{C}-23^{\circ} \mathrm{C}$; then $\mathrm{NEt}_{3}$ (2 equiv), 3,3-dimethylbutylamine ( 1.2 equiv), toluene, $23^{\circ} \mathrm{C}, 95 \%$ ( 2 steps); (b) 3-(4,4,5,5-tetramethyl-1,3,2-dioxaborolan-2-yl)pyridin-2-amine (1 equiv), X-Phos Pd G2 ( 0.05 equiv), X-Phos ( 0.05 equiv), $\mathrm{K}_{3} \mathrm{PO}_{4} \cdot \mathrm{H}_{2} \mathrm{O}$ ( 3 equiv), $\mathrm{THF} / \mathrm{H}_{2} \mathrm{O}$ (10:1), $80^{\circ} \mathrm{C}, 80 \%$; (c) 6-(2-aminopyridin-3-yl)-N-methylpyrimidin-4-amine (1.4 equiv), $\mathrm{Pd}_{2}(\mathrm{dba})_{3}$ (0.02 equiv), Xantphos (0.06 equiv), $\mathrm{Cs}_{2} \mathrm{CO}_{3}$ (1.4 equiv), 1,4-dioxane, $80^{\circ} \mathrm{C}, 61 \%$.

$$
\text { (2\% }
$$

(a) tert-butyl carbamate (1.2 equiv), X-Phos Pd G2 (0.05 equiv), X-Phos ( 0.10 equiv), $\mathrm{Cs}_{2} \mathrm{CO}_{3}$ (1.4 equiv), 1,4-dioxane, $110{ }^{\circ} \mathrm{C}$; then $\mathrm{HCl}$ in isopropanol, $23^{\circ} \mathrm{C}, 45 \%$ (2 steps); (b) methylamine hydrochloride (3.8 equiv), $\mathrm{K}_{2} \mathrm{CO}_{3}$ (2 equiv), DMSO, $90^{\circ} \mathrm{C}, 92 \%$; (c) diphenyl carbonate (1.15 equiv), DMAP (1.2 equiv), DMF, $100^{\circ} \mathrm{C}, 48 \%$; (d) 1-(5-amino-2-fluoro-4-methylphenyl)-3-(3,3-dimethylbutyl)urea (1 equiv), DMAP (1.1 equiv), DMF, $90^{\circ} \mathrm{C}, 14 \%$.

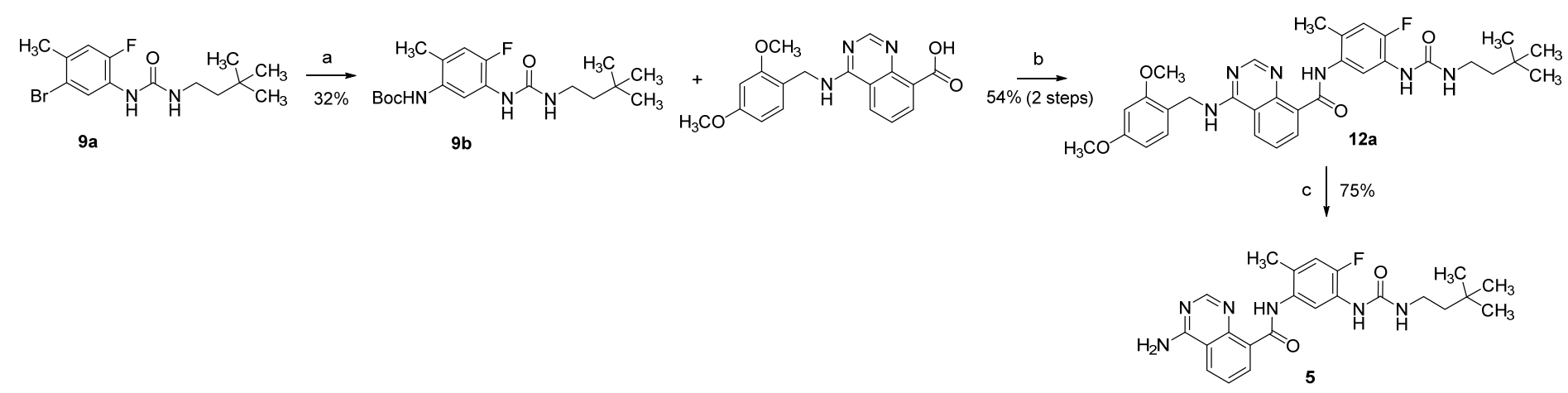

(a) tert-butyl carbamate $\left(2 \times 1.2\right.$ equiv), $\mathrm{Pd}_{2}$ (dba) 3 ( $2 \times 0.025$ equiv), Xantphos $\left(2 \times 0.06\right.$ equiv), $\mathrm{Cs}_{2} \mathrm{CO}_{3}\left(3\right.$ equiv), $\mathrm{THF}, 90^{\circ} \mathrm{C}-110^{\circ} \mathrm{C}, 32 \% ;(\mathrm{b}) \mathrm{TFA} / \mathrm{CH} \mathrm{Cl}_{2}(1: 1), 23^{\circ} \mathrm{C} ;$ then 4-((2,4-dimethoxybenzyl)amino)quinazoline-8-carboxylic acid (1 equiv), HATU (2 equiv), $\mathrm{NEt}_{3}$ ( 7 equiv), DMF, $23^{\circ} \mathrm{C}, 54 \%(2 \mathrm{steps}) ;(\mathrm{c}) \mathrm{TFA}^{\circ}, 70^{\circ} \mathrm{C}, 75 \%$. 
等<smiles>Cc1cc(F)c(NC(=O)NCCC(C)(C)C)cc1Br</smiles>

$\underset{80 \%(2 \text { steps })}{\stackrel{b}{\longrightarrow}}$

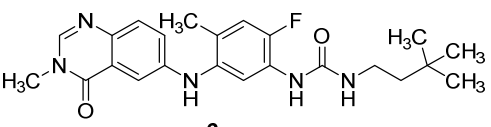

$9 a$

(a) tert-butyl carbamate (1.5 equiv), $\mathrm{Pd}_{2}$ (dba) $)_{3}$ (0.025 equiv), Xantphos ( 0.05 equiv), $\mathrm{Cs}_{2} \mathrm{CO}_{3}$ (2 equiv), THF, $80^{\circ} \mathrm{C}, 95 \%$; (b) TFA, $23^{\circ} \mathrm{C}$; then 1 - $(5$-bromo-2-fluoro-4methylphenyl)-3-(3,3-dimethylbutyl)urea (1 equiv), BrettPhos Pd G1 (0.05 equiv), BrettPhos ( 0.05 equiv), $\mathrm{NaOtBu}\left(4\right.$ equiv), $1,4-$ dioxane, $100{ }^{\circ} \mathrm{C}, 80 \%(2$ steps).
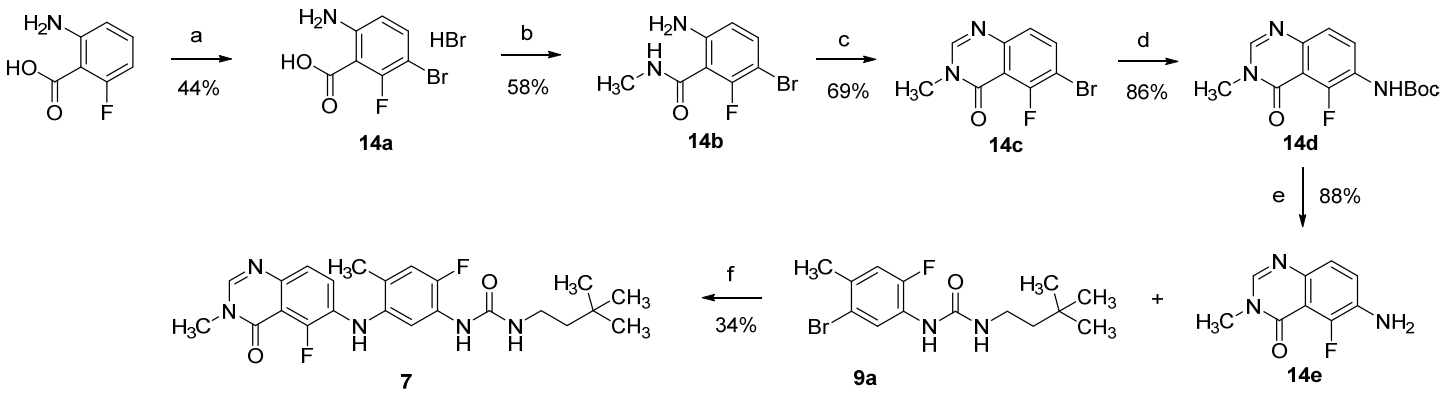

(a) bromine (1 equiv), $\mathrm{MeOH},-78^{\circ} \mathrm{C}, 44 \%$; (b) methylamine hydrochloride (5 equiv), $\mathrm{HATU}$ ( 2 equiv), $\mathrm{NEt}_{3}$ ( 10 equiv), $\mathrm{DMF}, 23^{\circ} \mathrm{C}, 58 \%$; (c) $4.0 \mathrm{M} \mathrm{HCl}$ in 1,4-dioxane/(EtO) ${ }_{3} \mathrm{CH}(1: 6), 110{ }^{\circ} \mathrm{C}, 69 \%$; (d) tert-butyl carbamate (1.2 equiv), $\mathrm{Pd}_{2}(\mathrm{dba})_{3}\left(0.02\right.$ equiv), Xantphos $\left(0.06\right.$ equiv), $\mathrm{Cs}_{2} \mathrm{CO}_{3}(1.2$ equiv), toluene $90{ }^{\circ} \mathrm{C}, 86 \%$; (e) $4.0 \mathrm{M} \mathrm{HCl}$ in 1,4-dioxane (12 equiv), EtOAc, $23^{\circ} \mathrm{C}, 88 \%$; (f) 1-(5-bromo-2-fluoro-4-methylphenyl)-3-(3,3-dimethylbutyl)urea (1 equiv), $\mathrm{Pd}(\mathrm{OAc})_{2}$ ( 0.05 equiv), Xantphos ( 0.05 equiv), $\mathrm{Cs}_{2} \mathrm{CO}_{3}$ ( 3 equiv), $3 \AA$ molecular sieves, 1,4 -dioxane, $100{ }^{\circ} \mathrm{C}, 34 \%$.

NMR Spectra and HPLC for GNE-0749 (7) 


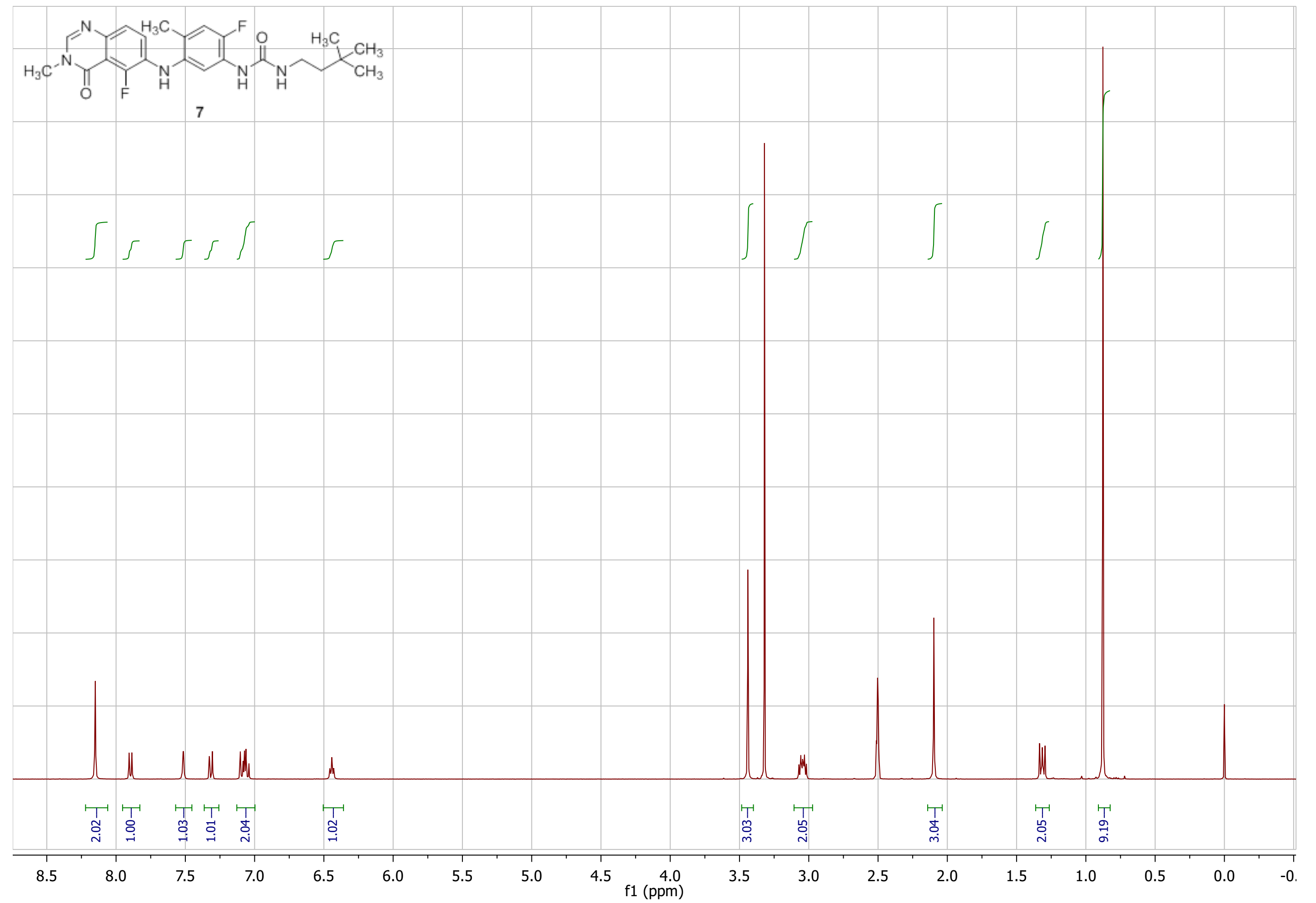




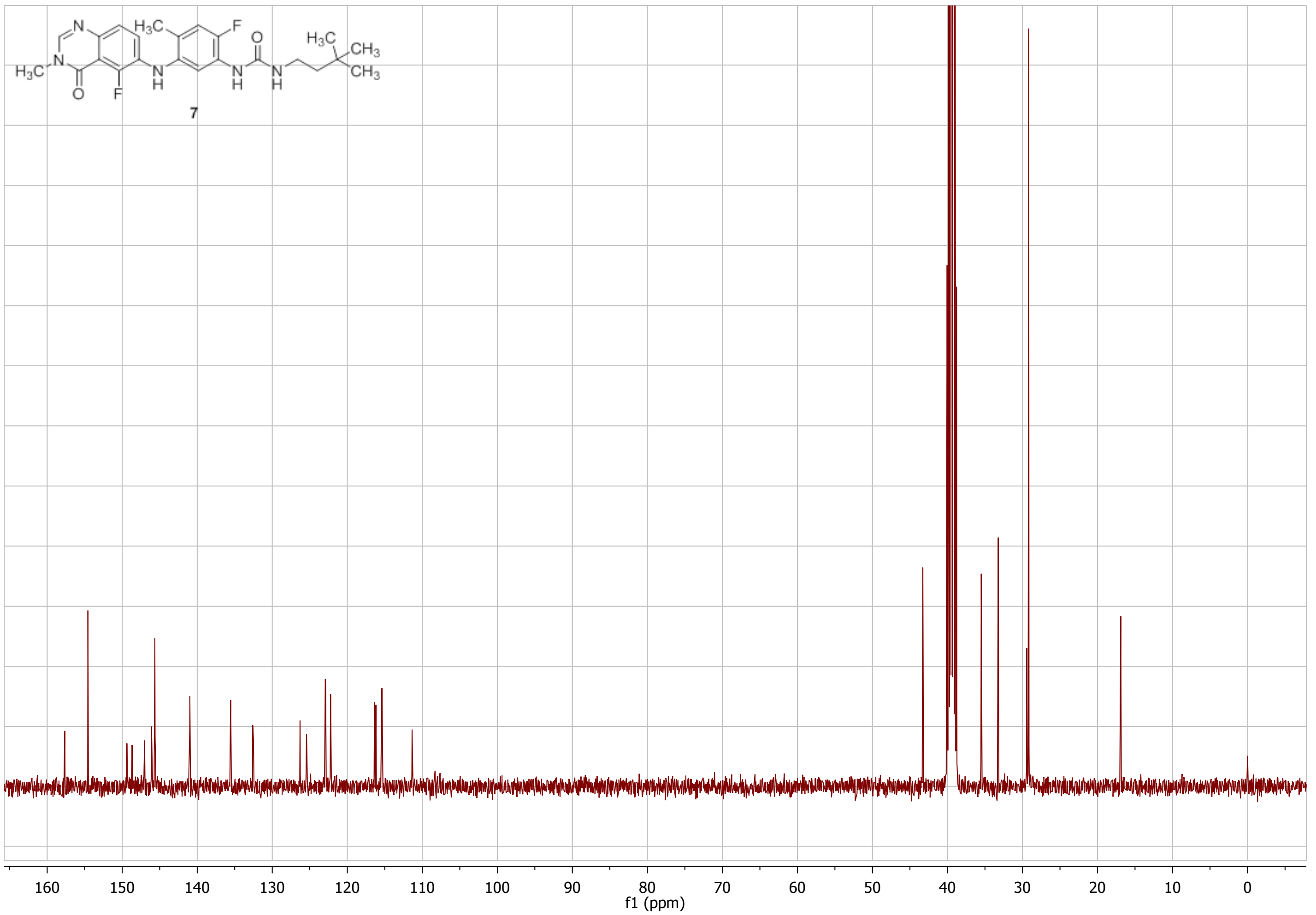




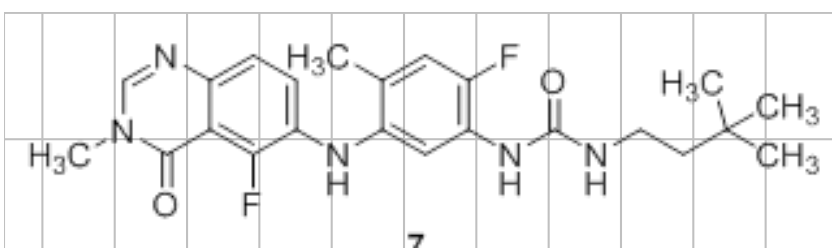


MS Report

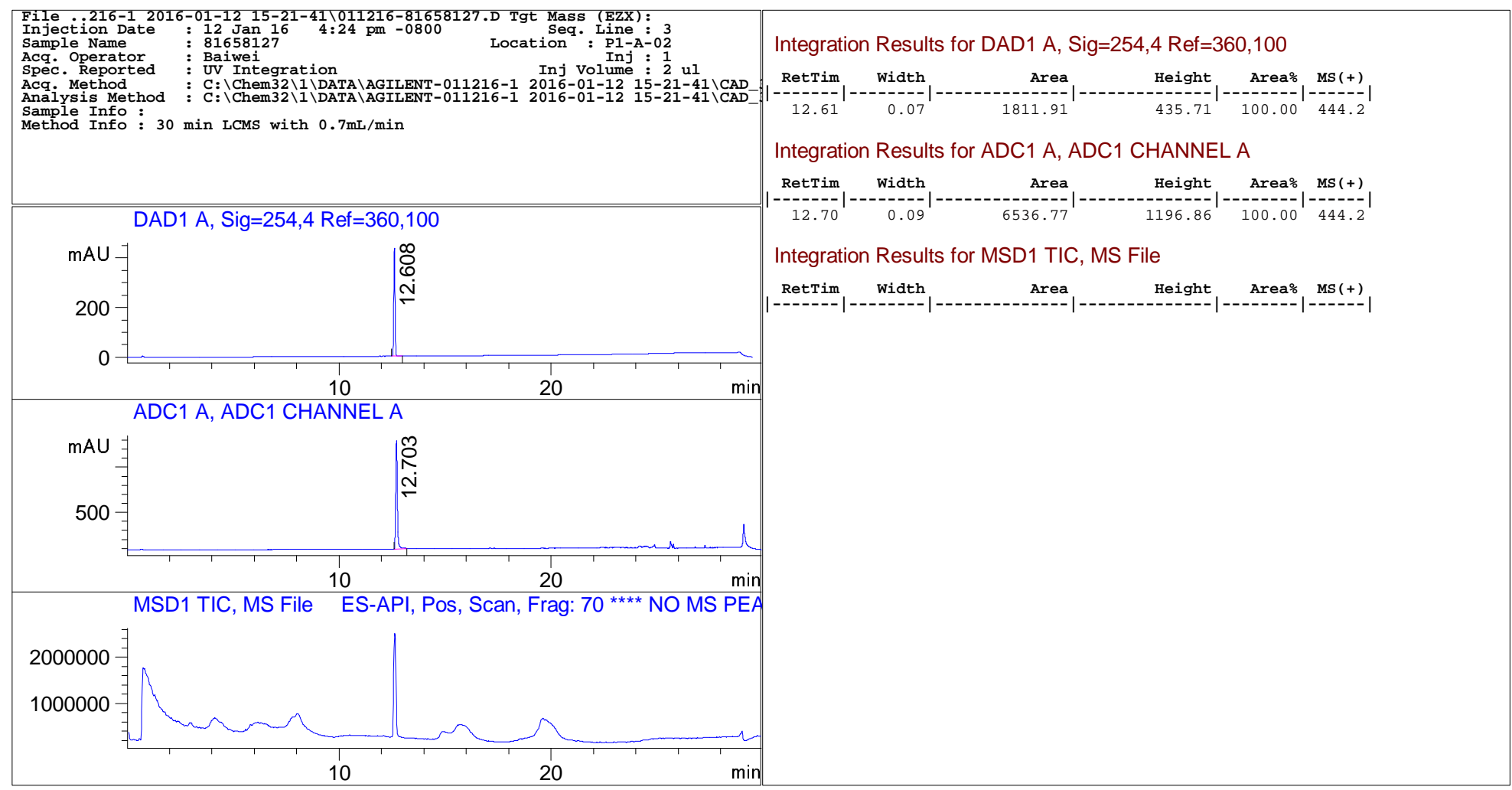

Ret. Time: 12.61 $<<<$ POSITIVE SPECTRA $>>>>$

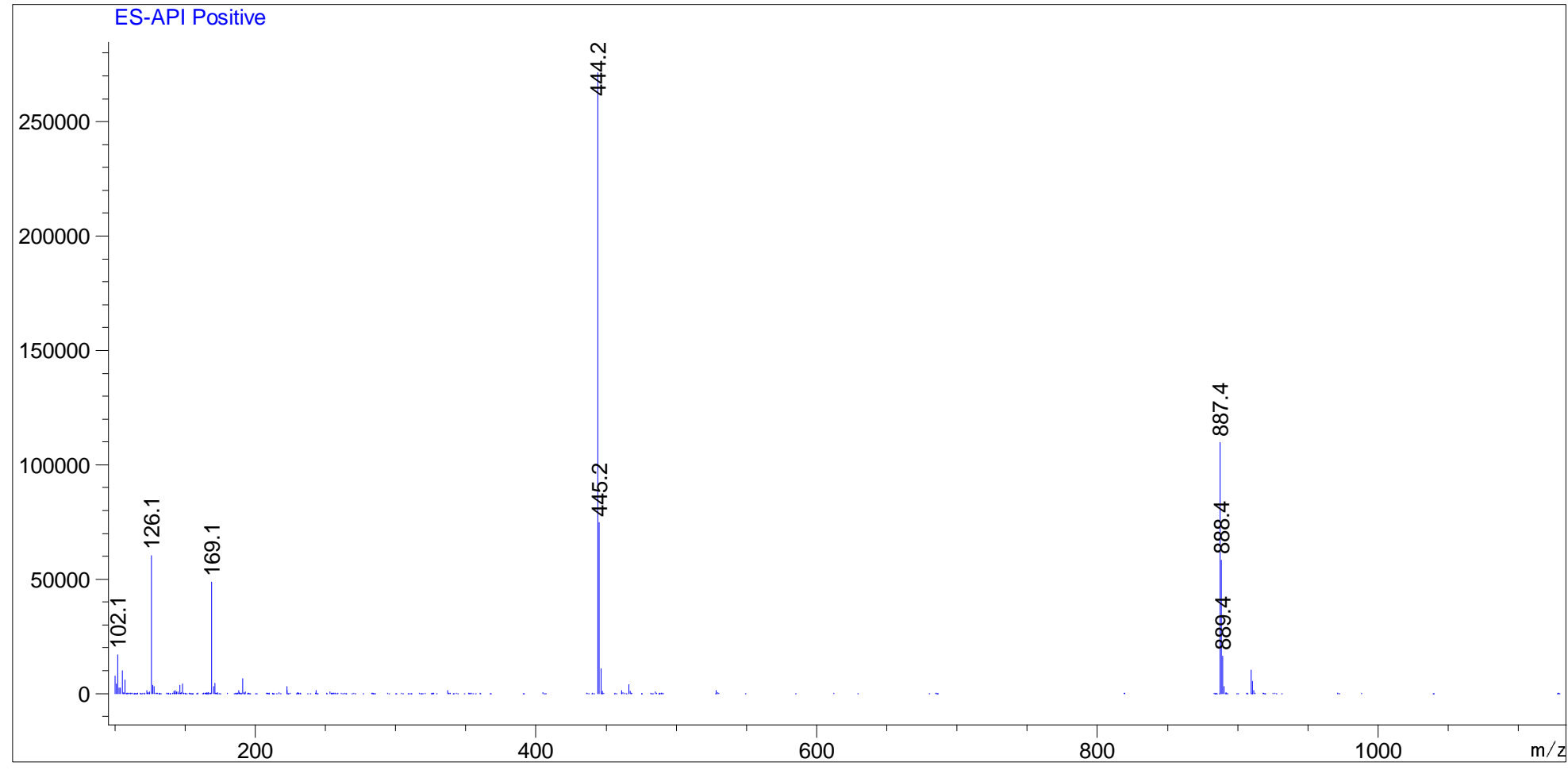

